\title{
Impact of binding to the Multidrug Resistant Regulator protein LmrR on the photo-physics and-chemistry of photosensitizers
}

\author{
Sara H. Mejías, ${ }^{*, a}$ Gerard Roelfes, ${ }^{a}$ and Wesley R. Browne*,a \\ Light activated photosensitizers generate reactive oxygen species (ROS) that interfere with cellular components and can induce \\ cell death, e.g., in photodynamic therapy (PDT). The effect of cellular components and especially proteins on the photochemistry \\ and photophysics of the sensitizers is a key aspect in drug design and the correlating cellular response with the generation of \\ specific ROS species. Here, we show the complex range of effects of binding of photosensitizer to a multidrug resistance protein, \\ produced by bacteria, on the formers reactivity. We show that recruitment of drug like molecules by LmrR (Lactococcal multidrug \\ resistance Regulator) modifies their photophysical properties and their capacity to induce oxidative stress especially in ${ }^{1} \mathrm{O}_{2}$ \\ generation, including rose bengal (RB), protoporphyrin IX (PpIX), bodipy, eosin Y (EY), riboflavin (RBF), and rhodamine (Rh6G). The \\ range of neutral and charged dyes with different exited redox potentials, are broadly representative of the dyes used in PDT.
}

\section{Introduction}

Photosensitizers (PS) that generate reactive oxygen species (ROS), primarily singlet oxygen $\left({ }^{1} \mathrm{O}_{2}\right)$ by triplet energy transfer, but also superoxide and hydroxyl radicals, are key tools in the study of damage to cellular components such as proteins, nucleic acids, or/and lipids caused by $\mathrm{ROS}^{1-4}$ as well as, in photodynamic therapy (PDT). ${ }^{5-8} \mathrm{~A}$ challenge encountered in the use of especially singlet oxygen photosensitizers in biological environments is the effect that cellular components have on their activity and the type and fate of the ROS species generated, especially in comparison to their photochemistry in, e.g., aqueous or organic media.

Organisms respond to reactive oxygen species (ROS) using redox active proteins through a set of reversible, e.g., with methionine (Met) and cysteine (Cys) and irreversible, oxidation of tryptophan (Trp) and tyrosine (Tyr) residues and the protein backbone. ${ }^{9}$ In addition to passive interference, bacterial cells trap 'drug-like' molecules for transport out of the cell using so called multidrug resistant (MDR) transporters. MDRs regulate removal of a wide variety of chemically distinct compounds from the cell, reducing the efficacy of drugs. ${ }^{10}$ The LmrR (Lactococcal multidrug resistance Regulator) protein, for example, is primarily responsible for the multidrug resistance in Lactococcus lactis bacteria. ${ }^{11-13}$ The LmrR protein used in this study is a drug resistance regulator in bacteria ${ }^{14,15}$, and although responsible for sensing foreign molecules and stimulating the transport system, the mechanism for transport out of cells involves binding of the foreign species within protein environments such as in LmrR. ${ }^{11,16,17}$

It is a homodimeric protein, which binds with relatively high affinities to, e.g., the DNA-binding compounds Hoechst 33342, daunomycin, ethidium bromide, and rhodamine $6 \mathrm{G}$, by sandwiching them between the tryptophan residues present within its hydrophobic pocket (Figure 1). ${ }^{11,18,19}$ LmrR's structure provides for a broad and shallow conformational energy surface in which the conformation can shift readily to accommodate

\footnotetext{
Stratingh Institute for Chemistry, University of Groningen, Nijenborgh 4, 9747 AG, Groningen, The Netherlands, w.r.browne@rug.nl.

Electronic Supplementary Information (ESI) available: experimental procedures, CD and thermal stability data, Raman spectra and additional cryo-electron micrographs
}

structurally unrelated compounds. ${ }^{19}$ Interaction of the protein LmrR with photoactive compounds, allows exploration of the effect of inclusion within proteins through, e.g., transition dipolee moments and energetics of bound molecules through the electric fields produced by charged amino acids and/or nonspecific interactions, ${ }^{20-23}$ on their photophysical and photochemical properties.
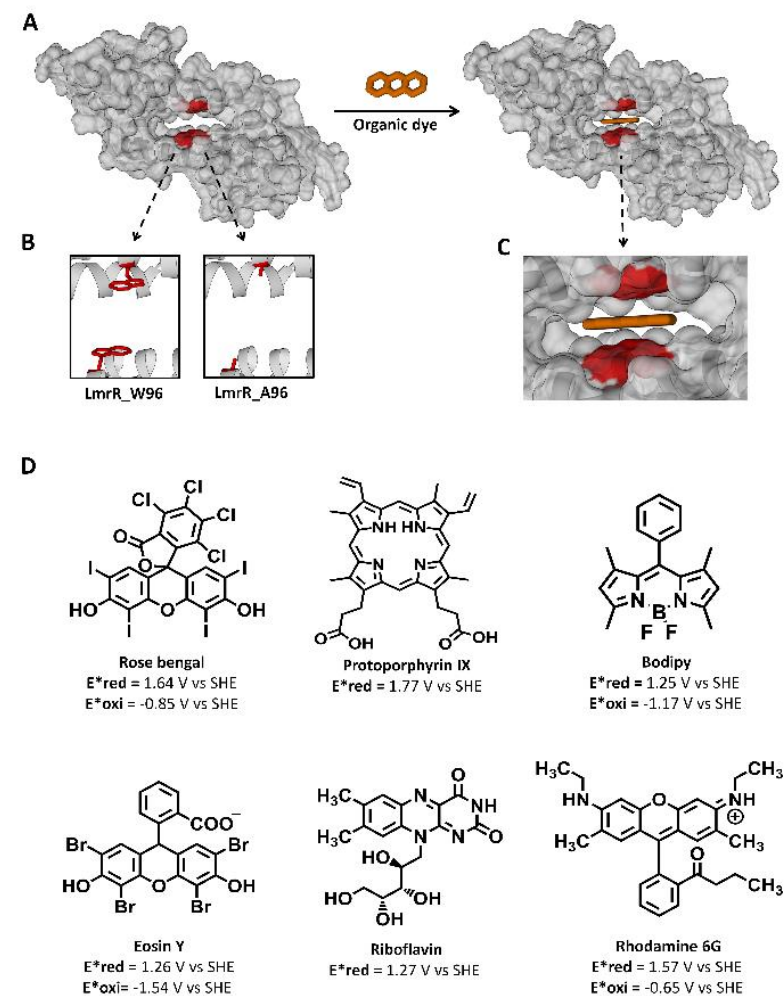

Figure 1. (A) Left, space filled representation of dimeric LmrR (pdb 3F8B) with an expansion of the hydrophobic pocket; right, same representation but with the organic dye filling the pocket. Organic dye is represented as three orange rings. (B) Cartoon representation of the LmrR W96 pocket with a tryptophan in the 96 position, left, and LmrR_A96 pocket with alanine at 96 position, right. (C) Zooming
of the dye filled LmrR pocket. (D) Photosenzitisers used in the current study with of the dye filled $\mathrm{LmrR}^{*}$ pocket. (D) Photosenzitisers used in the current study wit
their reduction ( ${ }^{*}{ }_{\text {red }}$ ) and oxidation ( $\mathrm{E}^{*}$ oxi) potential of the photoexcited state.

Here we make use the promiscuity of LmrR to bind small molecules to provide a platform on which to establish the effect of binding on the photophysical and photochemical properties 
of a wide range of commonly used ${ }^{3} \mathrm{O}_{2}$ sensitizers, including rose bengal (RB), protoporphyrin IX (PpIX), bodipy, eosin $Y(E Y)$, riboflavin (RBF), and rhodamine (Rh6G) (Figure 1D). ${ }^{24-30}$ The selected dyes are neutral or charged dyes with different exited state redox potentials and intersystem crossing quantum yields as a broad representation of the dyes used in PDT. The photophysical and chemical properties of the selected dyes are known to be strongly influenced by their environment. ${ }^{31-37}$ Predicting the changes that occur in the hydrophobic pocket of LmrR is challenging as changes in aromatic interactions (e.g., the interaction with tryptophans), the local environment, nature of the fluorophore (including the singlet and triplet lifetimes, redox potentials, photochemistry) each influence excited state properties. Hence, the range of dyes chosen reflects as much as possible the broad range of structures employed as ${ }^{1} \mathrm{O}_{2}$ generators. The affinity of LmrR for binding planar organic dyes (Figure 1C) and the effect that binding has on the photophysics including photostability of the dyes under conditions of continuous irradiation with visible light is explored. The generation of singlet oxygen is of particular interest. We show that the singlet oxygen generated in proximity of the protein undergoes rapid reaction with it, and that tryptophan provides sacrificial protection for the bound dye, extending the life of the dyes, in many cases, substantially.

\section{Results and discussion}

\section{Design, synthesis and characterization of LmrR-fluorophore complex.}

LmrR is homodimeric protein (molecular weight around $15 \mathrm{kDa}$ ) that contains a large hydrophobic pocket at its dimer interface and its conformation can shift readily to accommodate planar molecules (Figure 1A).11,12,19,38-40 Its high affinity for planar drugs is ascribed to two tryptophans at its dimer interface, position 96 (and 96'on the dimer partner). $11,12,19,38-40$ Two variants of $L m r R$ are used in the present study (Figure 1B, S1 and S2): LmrR_A96 where the tryptophans within the cavity (position 96) are substituted with alanine and LmrR_W96 where the tryptophans are retained. For both variants the other tryptophans present at positions 67 and 124 (on the strep tag) are substituted for alanine to avoid interference.
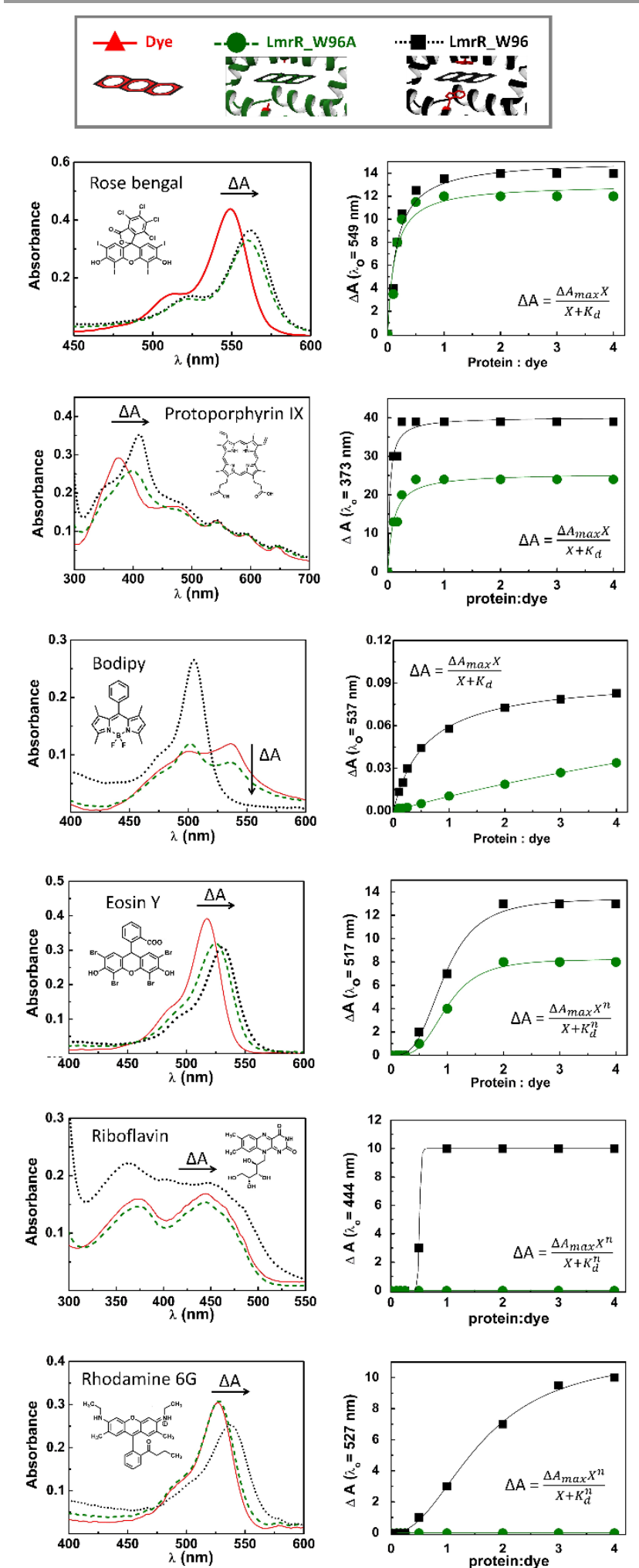

Figure 2. Left: UV-Vis absorption spectra of the dyes (red straight lines) LmrR_A96-dye assemblies (green dashed lines) and LmrR_W96-dye (black dotted line). Right: the red shift in absorbance maxima of the dyes used (indicated in the figure) upon serial addition of LmrR_W96A (green circles) and LmrR W96 (black squares). Fitting curves are shown in green and black for LmrR_A96 and
LmrR_W96, respectively.

The affinity of the dyes for each of the proteins was estimated by UV-Vis absorption spectroscopy. Their interaction with the LmrR proteins (W96 and A96) is manifested typically in a red 
shift in their visible absorbance compared to that in buffer (Figure 2, S4 and table S1), which is consistent with localization within the hydrophobic environment of the protein pocket (solvatochromism). ${ }^{31,32,35}$ The shift is greater when tryptophan is present indicating additional interactions with the tryptophan residues, e.g., through $\pi-\pi$ stacking. ${ }^{41-44}$ The shift in absorption maximum $\left(\Delta \lambda_{\max }\right)$ as the protein-dye ratio was varied was fitted (Figure 2) using a saturation growth model (equation 1) or Hill model (equation 2) where it provided a better fit to the data obtained. Equation 1 is used satisfactorily for rose bengal, protoporphyrin IX, and bodipy, while equation 2 was used for eosin $Y$, riboflavin and rhodamine $6 \mathrm{G}$ to yield the dissociation constant $\left(K_{d}\right)$ (table 1$)$.

$$
\begin{array}{ll}
\Delta A=\frac{\Delta A_{\max X}}{X+K_{d}} & (\text { Equation 1) } \\
\Delta A=\frac{\Delta A_{\max X^{n}}}{X+K_{d}^{n}} & (\text { Equation 2) }
\end{array}
$$

Table 1. $K_{d}, \Delta \lambda_{\max }$ and $n$ values for $L m r R$-dye interaction.

\begin{tabular}{|c|c|c|c|c|c|c|}
\hline & \multicolumn{2}{|c|}{$\mathrm{K}_{\mathrm{d}}(\mu \mathrm{M})$} & \multicolumn{2}{|c|}{$\Delta \lambda_{\max }(\mathrm{nm})$} & \multicolumn{2}{|c|}{$\mathrm{n}$} \\
\hline & A96 & W96 & A96 & W96 & A96 & W96 \\
\hline Protoporphyrin IX & 0.10 & 0.03 & 26 & 40 & 1 & 1 \\
\hline Rose bengal & 0.15 & 0.12 & 13 & 15 & 1 & 1 \\
\hline Bodipy & 13.7 & 0.59 & 0.1 & 0.1 & 1 & 1 \\
\hline Eosin Y & 0.99 & 0.94 & 8 & 13 & 3.5 & 3.2 \\
\hline Riboflavin & 0 & 0.51 & 0 & 10 & 0 & 34.6 \\
\hline Rhodamine 6G & 0 & 1.59 & 0 & 11.5 & 0 & 2.2 \\
\hline
\end{tabular}

Protoporphyrin IX shows the greatest affinity for the LmrR pocket with a $K_{d}$ of $30 \mathrm{nM}$ and $100 \mathrm{nM}$ for LmrR_W96 and LmrR_A96, respectively. Rose bengal and eosin $Y$ show similar affinity for both LmrR_W96 and LmrR_A96 indicating that they bind within the pocket due mainly to hydrophobic interactions. However, rose bengal binding can be fit well with a saturation model and binds more tightly ( $K_{d}$ of ca. $0.2 \mu \mathrm{M}$ ) to the pocket than eosin $Y\left(K_{d}\right.$ of ca. $\left.1 \mu \mathrm{M}\right)$, which showed Hill type binding. Bodipy shows only a minor shift in its maximum absorbance (3 $\mathrm{nm})$, consistent with the minor solvatochromic shift expected for bodipy dyes. ${ }^{45}$ Thus, the binding affinity was determined from the decrease in absorbance at $537 \mathrm{~nm}$ assigned to absorbance by bodipy aggregates in the absence of the protein. The binding affinity of bodipy to LmrR_W96 is much greater than to LmrR_A96 with a $K_{d}$ of $0.59 \mu \mathrm{M}$ for LmrR_W96 vs $K_{d}$ of 13.7 $\mu \mathrm{M}$ for LmrR_A96 indicating that aromatic interactions are important for this dye. Riboflavin and rhodamine 6G show changes in their spectra upon addition of LmrR_W96, but not with LmrR_W96A, indicating either that binding relies on interaction with the tryptophans or that the pocket itself has only a negligible electronic effect on the bound dyes. ${ }^{46}$ The absorption bands of the riboflavin bound to LmrR_W96 are slightly shifted and show more pronounced vibronic structure consistent with confinement of the photosensitizer within the protein pocket in the presence of tryptophan 47,48 showing a $K_{d}$ of $0.5 \mu \mathrm{M}$. Rhodamine $6 \mathrm{G}$ binds more weakly to LmrR_W96 with a $K_{d}$ of $1.59 \mu \mathrm{M}$. The structural stability of LmrR-dye complex was verified by circular dichroism (Figure S5), which confirms structure and thermal stability of $\mathrm{LmrR}$ is not affected upon binding of the dyes $\left(T_{m}=50^{\circ} \mathrm{C}\right)$.

Overall, although the tryptophan is not essential for dye binding, for most of the dyes the tryptophan residues increase binding affinity. Eosin $Y$, riboflavin and rhodamine show a Hill type response which indicates that a critical amount of protein is required to observe dye binding. Hence an excess of protein is required to ensure that essentially all dye is bound within the pocket of the LmrR protein and in the studies described below a 4:1 stoichiometry of protein:dye is used, unless stated otherwise.

Influence of LmrR on dye luminescence.

The photoluminescence of the dyes used here is sensitive to the hydrophobicity of their environment, conformational changes induced by binding and the presence of quenchers (of singlet and/or triplet excited states via electron or energy transfer). ${ }^{31,32,34,49}$ The effect of the hydrophobic environment and, moreover, tryptophan moieties on the emission is therefore useful in studying protein dye interactions (Figure 3, see also ESI Tables S9 and S10).

For rose bengal, the increase in emission upon binding either of the protein mutants is substantial and is consistent with the increase in lifetime observed typically in apolar and nonhydrogen bonding media. ${ }^{50,51}$ For eosin $Y$, rhodamine $6 G$, protoporphyrin IX and bodipy, the increase in emission upon binding to the protein is more pronounced with LmrR_W96 than with LmrR_W96A. Protoporphyrin IX aggregates in solution, which reduces the lifetime of the singlet-excited state and hence reduces its quantum yield of emission. ${ }^{52,53}$ Its emission increases slightly when bound to LmrR_A96, while a notable increase is observed with LmrR_W96, indicating that tryptophan units assist in breaking up of homo-aggregates. For bodipy the observed increase is attributed to a loss of rotational freedom at the meso position which is expected to contribute to the increase in emission. ${ }^{54,55-57}$ In contrast to the other dyes, riboflavin emission is heavily quenched by LmrR_W96, consistent with electron transfer between tryptophan and riboflavin. ${ }^{58-60}$ For all dyes, the Stokes shift is less with LmrR_A96 and LmrR_W96 than in buffer alone, which is consistent with confinement in the hydrophobic environment of the protein cavity and differences in solvent reorganization kinetics.

The emission from the tryptophan decreases concomitant with addition of the dyes with almost complete quenching with less than a 10-fold excess of each dye (Figure 4C, and S6). However, although the residual emission is weak, the emission lifetime does not change significantly compared to that of LmrR_W96 in the absence of the dye (Figure S7 and table S9 and $\mathrm{S} 10)$, indicating static quenching.

The emission decay lifetimes of the dyes in the absence and presence of the LmrR_W96 compared with that in non-aqueous solvents are consistent with incorporation with the hydrophobic pocket of the protein. For example, the fluorescence lifetime of protoporphyrin IX and bodipy increases in the presence of the protein when compared with the lifetime in water, consistent with the break-up of aggregates in the presence of the protein. For eosin $Y$ and rhodamine the 
fluorescence lifetime does not change significantly, while the emission intensity does which suggests amino acids increase fluorescence quantum yields. For riboflavin, measured lifetime is similar in the absence and presence of protein, while fluorescence intensity is decreased consistent with static quenching.
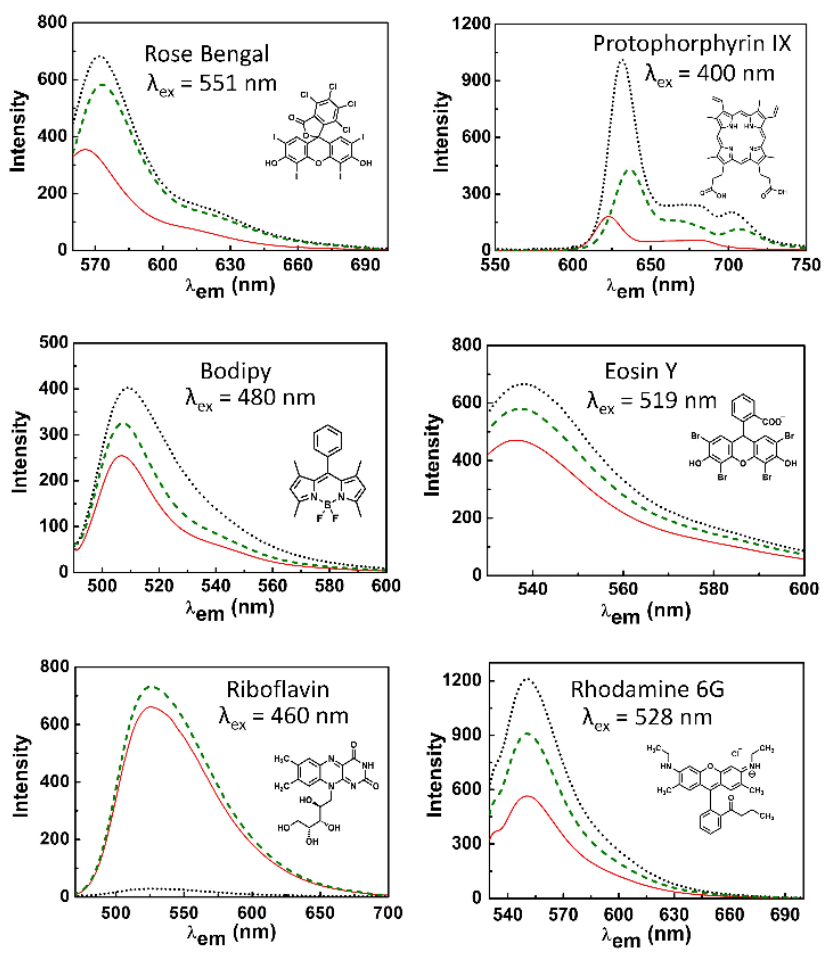

Figure 3. Absorbance corrected emission spectra of the photosensitizer excess of LmrR W96A (green dashed line), or LmrR W96 (black dotted line).

\section{Singlet oxygen $\left({ }^{1} \mathrm{O}_{2}\right)$ emission.}

The near-IR phosphorescence of ${ }^{1} \mathrm{O}_{2}$ upon irradiation of riboflavin in $\mathrm{D}_{2} \mathrm{O}$ is substantially diminished in the presence of LmrR_A96 and it is not detectable in the presence of the LmrR_W96 mutant (Figure 4A). Furthermore, continuous irradiation of riboflavin in $\mathrm{D}_{2} \mathrm{O}$, in the absence of protein, results in a decrease in ${ }^{1} \mathrm{O}_{2}$ emission intensity over time (ca. $20 \%$ over $5 \mathrm{~min}$, Figure 4B), due to attack of the ${ }^{1} \mathrm{O}_{2}$ generated on the dye. The loss of ${ }^{1} \mathrm{O}_{2}$ emission indicates that both $\mathrm{LmrR}$ variants either inhibit ${ }^{3} \mathrm{O}_{2}$ sensitization by quenching the triplet state of the riboflavin and/or the ${ }^{1} \mathrm{O}_{2}$ produced reacts rapidly, with the amino acids, such as the tyrosine residues present in the protein, and hence emission is quenched. ${ }^{61-64}$ Notably, ${ }^{1} \mathrm{O}_{2}$ emission in the presence of $\left[\mathrm{Ru}(\mathrm{bpy})_{3}\right]^{2+}$ is quenched completely in the presence of either protein (Figure S8.B). Since the dication $\left[\mathrm{Ru}(\mathrm{bpy})_{3}\right]^{2+}$ is unlikely to bind within the pocket, but instead to the anionically charged surface of the protein, its emission (at $610 \mathrm{~nm}$ ) is relatively unaffected by the proteins (Figure S8A). Hence it is clear that ${ }^{1} \mathrm{O}_{2}$, if formed, is in sufficient proximity to react rapidly with the protein. Hence, the absence of ${ }^{1} \mathrm{O}_{2}$ emission in the presence of the $\mathrm{LmrR}$ variants cannot be taken as a conclusive evidence for inhibition of ${ }^{1} \mathrm{O}_{2}$ generation.
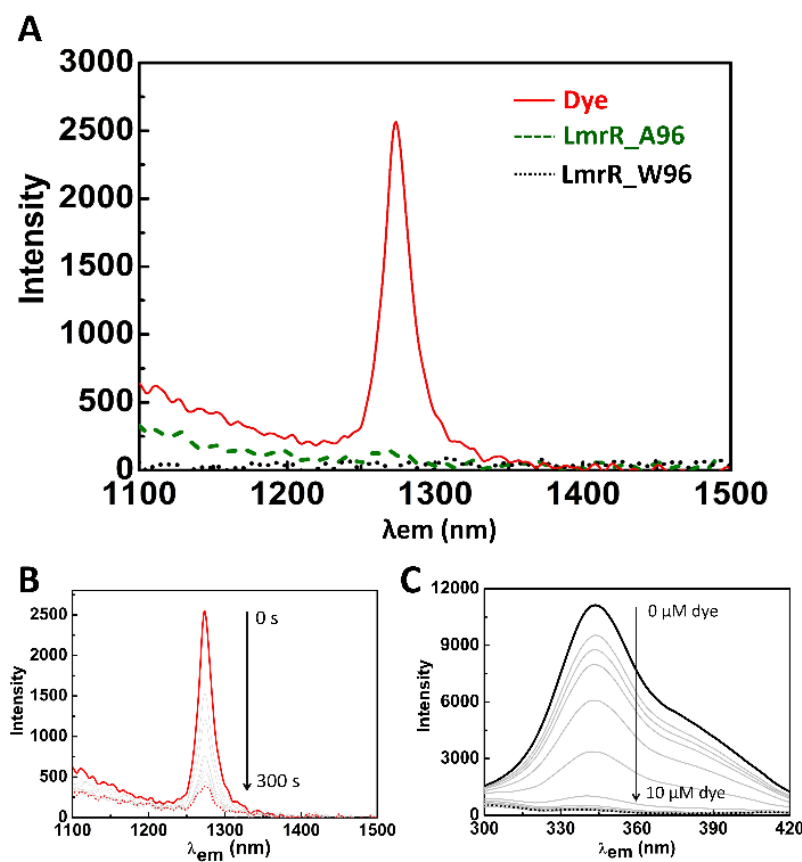

Figure 4. (A) NIR phosphorescence from ${ }^{1} \mathrm{O}_{2}$ generated upon irradiation of riboflavin in $\mathrm{D}_{2} \mathrm{O}$ (phosphate buffer $\mathrm{pD} 8$ ) (red straight line), in the presence of LmrR W96A (green dashed line), and in the presence of LmrR W96 (black dotted line). (B) Change in $\mathrm{NIR}$ phosphorescence over time under constant excitation $\left(\lambda_{\text {exc }}\right.$
$=455 \mathrm{~nm}$ ) of riboflavin in $\mathrm{D}_{2} \mathrm{O}$ (phosphate buffer $\mathrm{pD} 8$ ). $0 \mathrm{~s}$ (red straight line) to $=455 \mathrm{~nm}$ ) of riboflavin in $\mathrm{D}_{2} \mathrm{O}$ (phosphate buffer $\left.\mathrm{pD} 8\right)$. O $\mathrm{s}$ (red straight line) to
$300 \mathrm{~s}$ (red dotted line). (C) Tryptophan fluorescence $\left(\lambda_{\text {ex }} 280 \mathrm{~nm}\right.$ ) of $1 \mu \mathrm{\mu M}$ LmrR_W96 with increasing concentration of rose bengal $(0 \mu \mathrm{MM}-$ thick line, to 10 $\mu \mathrm{M}$ - dotted line).

\section{Photochemical stability of dyes.}

The bleaching of most of the dyes upon irradiation in the presence of $\mathrm{O}_{2}$ follows first order decay with rates of $2.4 \times 10^{-3}, 2.7 \times 10^{-3}$, and $3.6 \times 10^{-3}$ for rose bengal, bodipy and eosin $Y$, respectively (table 2 ). Riboflavin shows biexponential decay with a fast and slow component $(3.7 \times$ $10^{-2}$ and $8.1 \times 10^{-4}$, respectively), which indicates several degradation pathways in the presence of oxygen. Protoporphyrin IX and rhodamine $6 \mathrm{G}$ are stable under irradiation due to absence of ROS generation, attributed to formation of dye aggregates that quench the excited state (vide supra) of the dye for protoporphyrin $I^{65,66}$ and a low intersystem crossing quantum yield for rhodamine 6G.67,68 In the absence of oxygen, generally bleaching is slower (table 2). Excited state reduction $\left(E_{r e d}^{*}\right)$ and oxidation $\left(E_{o x}^{*}\right)$ potentials (Figure 1 and table S3) were calculated using the equation for Gibbs free energy of photoinduced electron transfer (see methods for details). ${ }^{69}$ The redox potential for tryptophan (1.09 V) ${ }^{70}$ is sufficiently low to allow for oxidation by the dyes. Most of the dyes show photobleaching in the absence of protein due to ROS generation (vide supra), and hence the observed behaviour of the dyes in the presence of protein is a combination of several interactions between dye-protein-ROS, the net result of which is determined by the thermodynamics and the kinetics of the interactions. Hence prediction of the behaviour of each dye in the presence of the LmrR is difficult and each dye needs to be studied separately. The effect of protein hydrophobicity and tryptophan on the photostability of the dyes was explored by monitoring their visible absorbance 
over time during extended irradiation in the presence and absence of protein and $\mathrm{O}_{2}$ (Figure 5 to Figure 7 and Figure $\mathrm{S} 9$ to S11). An increase in absorbance between 300 to $350 \mathrm{~nm}$ in the presence of both $\mathrm{O}_{2}$ and $\mathrm{LmrR}$ protein is attributed to the oxidation of amino acids (e.g., tyrosine and tryptophan) upon reaction with ${ }^{1} \mathrm{O}_{2} \cdot{ }^{61-64,71-73}$

In all cases, oxidative damage to the protein is not significant in the absence of $\mathrm{O}_{2}$ and hence excited state electron transfer (photoredox chemistry) from or to the dyes can be excluded. The time dependence of the changes in visible absorbance of each dye and LmrR-dye assemblies in the presence and absence of oxygen were fitted to decay models that fit best the data obtained, and hence these models do not, a priori, have physical meaning. It should be noted that in all cases the irradiation wavelength and intensity (ca. $300 \mathrm{~mW}$ ) was identical, and hence the photokinetic factors are comparable and all rate constants described below are relative and allow for broad comparison of the behaviours of the different classes of dye.

Table 2. Dye photobleaching kinetics.

\begin{tabular}{|c|c|c|c|c|c|c|c|c|}
\hline & \multicolumn{5}{|c|}{$\begin{array}{c}\text { Air equilibrated } \\
Y=A_{1} \exp \left(-k_{1} x\right)+A_{2} \exp \left(-k_{2} x\right)+Y_{0}\end{array}$} & \multicolumn{3}{|c|}{$\begin{array}{c}\text { Argon purged } \\
Y=A \exp (-k x)+Y_{0}\end{array}$} \\
\hline & $\begin{array}{c}k_{1} \\
\left(\mathrm{~s}^{-1}\right) \\
\times 10^{-3} \\
\end{array}$ & $\begin{array}{c}\mathrm{k}_{2} \\
\left(\mathrm{~s}^{-1}\right) \\
\times 10^{-3} \\
\end{array}$ & $A_{1}$ & $A_{2}$ & $Y_{0}$ & $\begin{array}{c}k \\
\left(s^{-1}\right) \\
\times 10^{-3} \\
\end{array}$ & A & $Y_{0}$ \\
\hline $\begin{array}{c}\text { Rose } \\
\text { bengal }\end{array}$ & 2.4 & - & 0.8 & - & 0.2 & 2.6 & 0.6 & 0.3 \\
\hline Bodipy & 2.7 & - & 1 & - & 0 & - & - & - \\
\hline Eosin $Y$ & 3.6 & - & 0.9 & - & 0.1 & 16 & 0.8 & 0.2 \\
\hline Riboflavin & 37 & 0.81 & 0.6 & 0.4 & 0 & 140 & 0.9 & 0.08 \\
\hline
\end{tabular}

The five arbitrary models used for fitting the bleaching data: linear decay (equation 3), mono exponential decay (equation 4), biexponential decay (equation 5), algebraic decay (equation 6) and stretched algebraic decay (equation 7).

$Y=Y_{o}-K x$

(equation 3)

$Y=Y_{o}+A e^{-k x}$

(equation 4)

$Y=Y_{o}+A_{1} e^{-k_{1} x}+A_{2} e^{-k_{2} x}$

(equation 5)

$Y=Y_{o}+\frac{A}{1+k x}$

(equation 6)

$Y=Y_{o}+\frac{A}{1+(k x)^{\beta}}$

(equation 7)

Fitting of the data (table S4 to table S9) provides apparent rate constants $(k)$ allowing comparison of the effect of the protein hydrophobicity and the tryptophans on dye photobleaching on the decay of absorbance. Overall, dye photobleaching is influenced by tryptophan except for rose bengal and rhodamine $6 \mathrm{G}$. Hence the dyes can be divided into three broad classes according to the effect of the LmrR protein: 1 . photobleaching is affected by protein hydrophobicity but tryptophan does not influence the decay rate; 2 . photobleaching is affected by the presence of tryptophan; 3 . photobleaching is essentially unaffected by the protein.
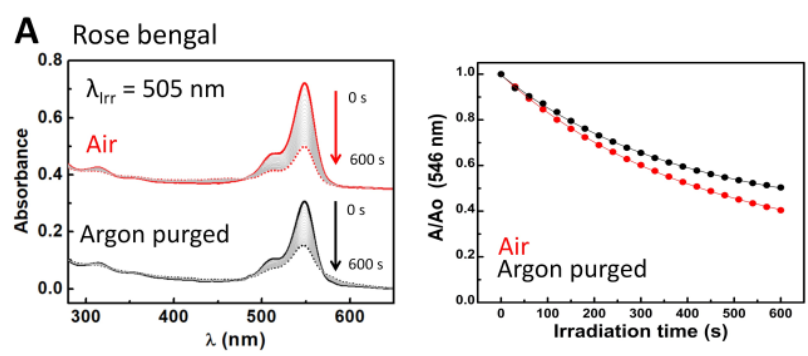

B A96_Rose bengal
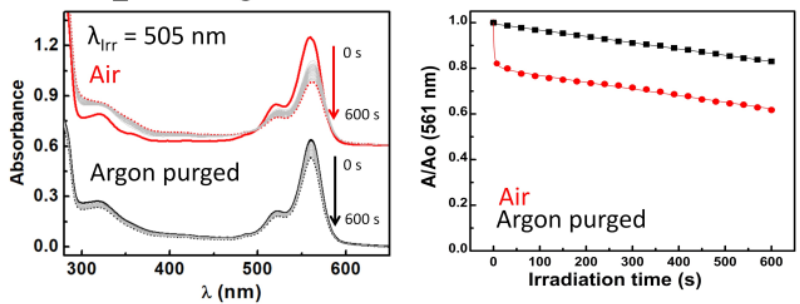

C W96_Rose bengal
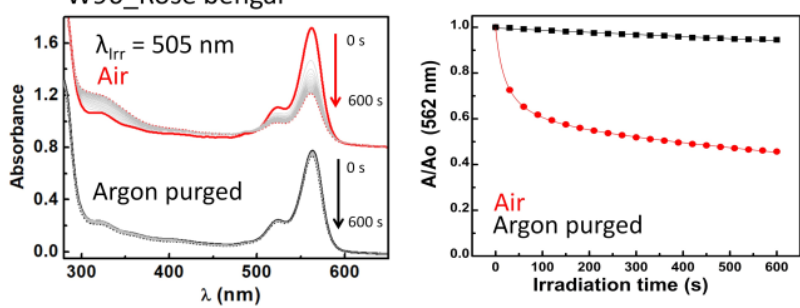

Figure 5. (Right) Change in absorbance upon irradiation of rose bengal over $600 \mathrm{~s}$ in the presence (red) and in absence (black) of $\mathrm{O}_{2}$ in solution (A), in the presence of LmrR_A96 (B) and in the presence of LmrR_W96 (C). Initial spectrum is a straigh line, final spectrum is a dashed line. Grey lines are spectra recorded at $30 \mathrm{~s}$ intervals. (Left) Absorbance at the visible absorbance maximum over time relative to initial absorbance in the presence (red) and absence of $\mathrm{O}_{2}$ (black). Straight line bengal in $50 \mathrm{mM}$ potassium phosphate buffer at $\mathrm{pD} 8$ prepared in $\mathrm{D}_{2} \mathrm{O}$. LmrR_A96 and LmrR_W96 were both $24 \mu \mathrm{M}$ (1:4 dye:protein ratio).

Rose bengal (Figure 5) represents the first class as its absorbance undergoes monoexponential decay upon irradiation that is unaffected by $\mathrm{O}_{2}, \mathrm{k}=2.4 \times 10^{-3} \mathrm{~s}^{-1}$ and $2.6 \times 10^{-}$ ${ }^{3} \mathrm{~s}^{-1}$, respectively. In the absence of oxygen, the presence of LmrR_A96 or W96, results in a large reduction in the rate of decay, and a change to linear decay $\left(\mathrm{k}=2.8 \times 10^{-4} \mathrm{~s}^{-1}\right.$ and $9.3 \times 10^{-}$ $5 \mathrm{~s}^{-1}$, respectively). The large decrease in rate suggests the protein shuts down the pathway observed when the dye is in solution in the absence of oxygen. Possibly, the protein's hydrophobic pocket limits the formation of hydroxyl radicals that are otherwise formed in water upon photoexcitation of rose bengal, ${ }^{74,75}$ and is consistent with the increase in the fluorescence observed also (vide supra). When oxygen is present, the protein-dye complex shows faster photobleaching than with the dye alone in solution. For both proteins, the decay can only be fit by a combination of linear and algebraic decay models (equation 3 and 6). For the algebraic part, the decay rate is $0.9 \mathrm{~s}^{-1}$ with LmrR_A96 and slower, $0.1 \mathrm{~s}^{-1}$, for LmrR_W96. A second linear decay component is required to fit the decay fully with a rate similar to that observed in the absence of oxygen (Figure 5 and table S4). 

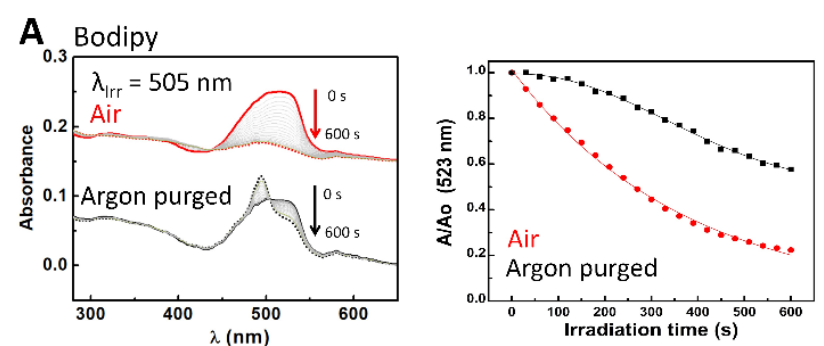

B A96_Bodipy
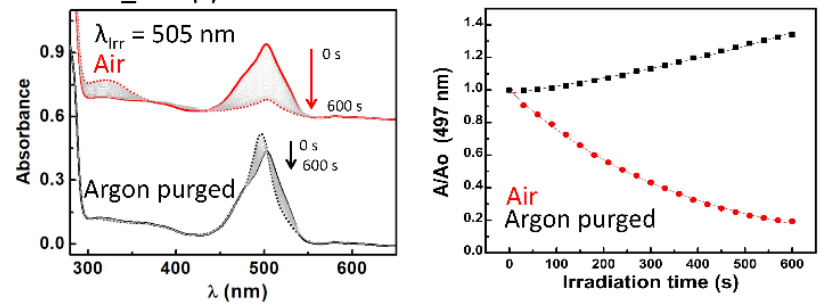

C W96_Bodipy
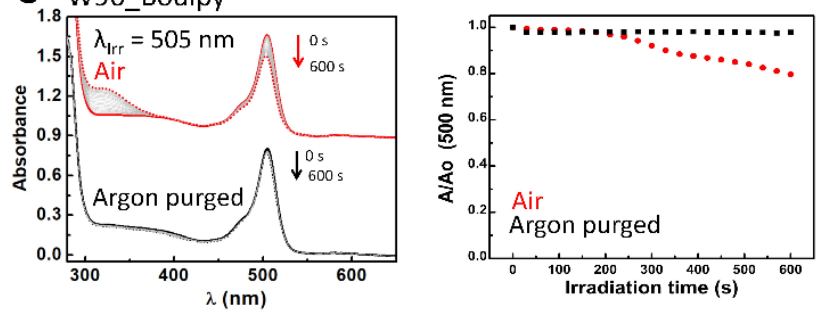

Figure 6. (Right) Change in absorbance upon irradiation of bodipy over $600 \mathrm{~s}$ in the presence (red) and in absence (black) of $\mathrm{O}_{2}$ in solution (A), in the presence of $\mathrm{LmrR} A 96(\mathrm{~B})$ and in the presence of LmrR W96 (C). Initial spectrum is a straight
line, final spectrum is a dashed line. Grey lines are spectra recorded at $30 \mathrm{~s}$ intervals. (Left) Absorbance at the visible absorbance maximum over time relative to initial absorbance in the presence (red) and absence of $\mathrm{O}_{2}$ (black). Straight line (black and red) is the fitting of the used model (see text). Conditions: $10 \mu \mathrm{M}$ bodipy in $50 \mathrm{mM}$ potassium phosphate buffer at pD 8 prepar
$\mathrm{LmrR}$ W96 were both $40 \mu \mathrm{M}$ (1:4 dye:protein ratio).

The second class includes most of the dyes, bodipy (Figure 6), eosin Y (Figure S9), protoporphyrin IX (Figure S10) and riboflavin (Figure S11). For these dyes, the hydrophobicity of the protein pocket has little influence on the decay observed, in contrast to the effect of the tryptophan. Notably each dye is affected by LmrR_W96 in a different manner, which is discussed below.

Bodipy shows monoexponential decay upon visible irradiation in the presence of oxygen $\left(2.7 \times 10^{-3} \mathrm{~s}^{-1}\right)$ (Figure 6). In absence of oxygen, a change in spectral shape is observed indicating changes in the bodipy probably due to ROS, however the change in aggregation state may also be a contributing factor. Similar changes are observed in the presence of LmrR_A96: exponential decay $\left(2.7 \times 10^{-3} \mathrm{~s}^{-1}\right)$ in the presence of oxygen and a spectral shift in the absence of oxygen. The presence of tryptophan inhibits the photodegradation substantially even in the presence of oxygen, however it is notable that protein degradation occurs instead (manifested in an increase in absorbance at ca. $350 \mathrm{~nm}$, Figure 6 and table S5). The rate of decay of the visible absorbance of eosin Y (Figure S9 and table S6) is similar to that of bodipy, but surprisingly, the decay rate in the absence of oxygen $16 \times 10^{-3} \mathrm{~s}^{-1}$ is faster than in the presence of oxygen $\left(3.6 \times 10^{-3} \mathrm{~s}^{-1}\right)$, indicating interaction of the dye with oxygen (quenching) is competing with an intramolecular decay pathway. The same trend is observed in the presence of LmrR_A96, $\left(5.6 \times 10^{-3} \mathrm{~s}^{-1}\right.$ and $1.1 \times 10^{-2} \mathrm{~s}^{-1}$, respectively). With LmrR_W96 monoexponential decay $\left(5.3 \times 10^{-}\right.$ $\left.{ }^{3} \mathrm{~s}^{-1}\right)$ is observed in presence of oxygen, however, in its absence, the decay follows an algebraic stretched decay $\left(3.3 \times 10^{-3} \mathrm{~s}^{-1}\right)$, which indicates, as for rose bengal, several pathways to dye degradation.

Protoporphyrin IX (Figure S10 and table S7), in the presence of oxygen, shows similar behaviour as bodipy in that upon irradiation in the presence of $L m r R \_A 96$ a shift in absorbance is observed with a monoexponential decay $\left(1.8 \times 10^{-3} \mathrm{~s}^{-1}\right)$. However, in contrast to bodipy, tryptophan accelerates the photodegradation of protoporphyrin IX in the presence of oxygen and required fitting with a biexponential model $(5.8 \times 10$ 2 and $\left.4.1 \times 10^{-3} \mathrm{~s}^{-1}\right)$. In the absence of oxygen, photobleaching is not observed in presence of both LmrR_A96 and LmrR_W96. In the absence of protein protoporphyrin does not undergo photodegradation upon irradiation which is consistent with aggregation induced excited state quenching (vide supra). ${ }^{76}$ Riboflavin (Figure S11 and table S8) is the only dye which shows quenching of its emission in the presence of tryptophan. The photodegradation pathways of riboflavin have been studied elsewhere. ${ }^{77-80}$ The photoreactivity of riboflavin arises from its triplet excited state. The absorbance of riboflavin decays monoexponentially $\left(0.14 \mathrm{~s}^{-1}\right)$ when irradiated in absence of oxygen. LmrR_A96 does not affect the decay substantially $(0.08$ $\mathrm{s}^{-1}$ ). By contrast, oxygen reduces the rate of bleaching with a biexponential decay observed $\left(3.7 \times 10^{-2} \mathrm{~s}^{-1}\right.$ and $\left.8.1 \times 10^{-4} \mathrm{~s}^{-1}\right)$, ascribed to additional reaction pathways including ${ }^{1} \mathrm{O}_{2}$ generation. In the presence of oxygen and LmrR_A96, a single exponential decay $\left(5.1 \times 10^{-2} \mathrm{~s}^{-1}\right)$ is observed, indicating that the protein interferes with the riboflavin ${ }^{1} \mathrm{O}_{2}$ decay pathway, consistent with the increase in absorbance at ca. $310 \mathrm{~nm}$ attributed to irreversible amino acid oxidation. LmrR_W96 inhibits all photobleaching in the absence of oxygen, ascribed to electron transfer deactivation between the riboflavin anion radical ( $\mathrm{RF}^{-}-$) and tryptophan cation radical $\left(\operatorname{Trp}(\mathrm{H})^{\circ}+\right)^{81} \mathrm{O}_{2}$ can compete with this pathway to form $\mathrm{O}_{2}{ }^{\circ}$ which reacts with the tryptophan radical irreversibly manifested again in an increase in absorbance at ca. $310 \mathrm{~nm}$. For rhodamine 6G (Figure 7 and table S9), which does not generate ${ }^{1} \mathrm{O}_{2}$ neither the protein and the presence of tryptophan affect significantly the decay trend or rate. Rhodamine $6 \mathrm{G}$ is photostable even in the presence of oxygen, consistent with its low triplet state quantum yield. ${ }^{82}$ Thus, neither degradation or oxidation is observed in the absence and the presence of the protein or oxygen. 

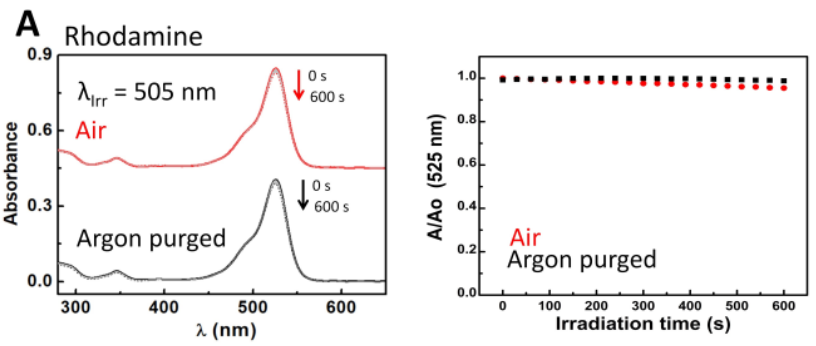

B A96_Rhodamine
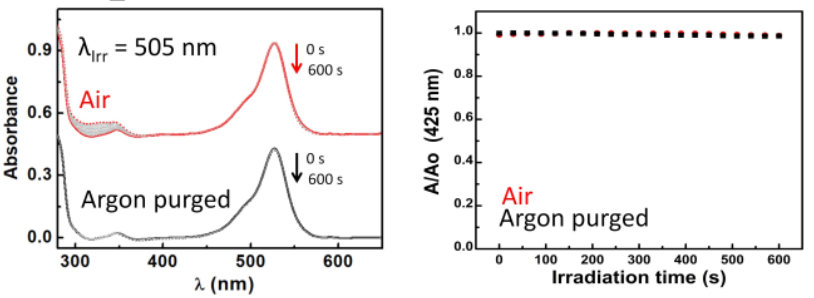

C W96_Rhodamine
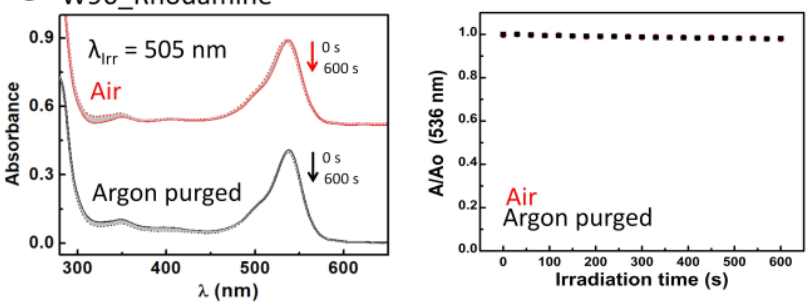

Figure 7. (Right) Change in absorbance upon irradiation of rhodamine $6 \mathrm{G}$ over $600 \mathrm{~s}$ in the presence (red) and in absence (black) of $\mathrm{O}_{2}$ in solution (A), in the
presence of LmrR A96 (B) and in the presence of LmrR W 96 (C). Initial spectrum is a straight line, final spectrum is a dashed line. Grey lines are spectra recorded at
$30 \mathrm{~s}$ intervals. (Left) Absorbance at the visible absorbance maximum over time relative to initial absorbance in the presence (red) and absence of $\mathrm{O}_{2}$ (black) Straight line (black and red) is the fitting of the used model (see text). Conditions: $6 \mu \mathrm{M}$ rhodamine $6 \mathrm{G}$ in $50 \mathrm{mM}$ potassium phosphate buffer at $\mathrm{pD} 8$ prepared in $\mathrm{D}_{2} \mathrm{O}$. LmrR_A96 and LmrR_W96 were both $24 \mu \mathrm{M}$ (1:4 dye:protein ratio).

Summary. The hydrophobic pocket of the LmrR protein is used to study the effect of protein hydrophobicity and tryptophan on the photostability of organic dyes under extended irradiation in the presence and absence of oxygen. LmrR shows high promiscuity for planar organic dyes with different structures and wide range of redox potentials. The dyes show high affinity for binding to the LmrR pocket with $K_{d}$ from nano to micromolar range. Upon interaction with LmrR, most of the dyes show changes in the absorption spectrum consistent with changes in the hydrophobicity due to the protein pocket and further changes in the presence of tryptophan that suggest additional electronic interactions between tryptophan and fluorophores. The selected dyes can sensitize the ROS (e.g., ${ }^{1} \mathrm{O}_{2}$ ) generation that leads to photodegradation of the dyes in solution. For example, with riboflavin ${ }^{1} \mathrm{O}_{2}$ emission decays over time due to dye bleaching. The presence of both LmrR_A96 or LmrR_W96, results in a near complete quenching of the ${ }^{1} \mathrm{O}_{2}$ emission, indicating that either the protein quenches the triplet state of the dye preventing ${ }^{1} \mathrm{O}_{2}$ generation or protein amino acids react with ${ }^{1} \mathrm{O}_{2}$ and hence preventing ${ }^{1} \mathrm{O}_{2}$ emission (Figure 8). Interestingly, tryptophan offers sacrificial protection to oxidation for most of the dyes, and in the case of bodipy prevents any degradation even in the presence of oxygen.

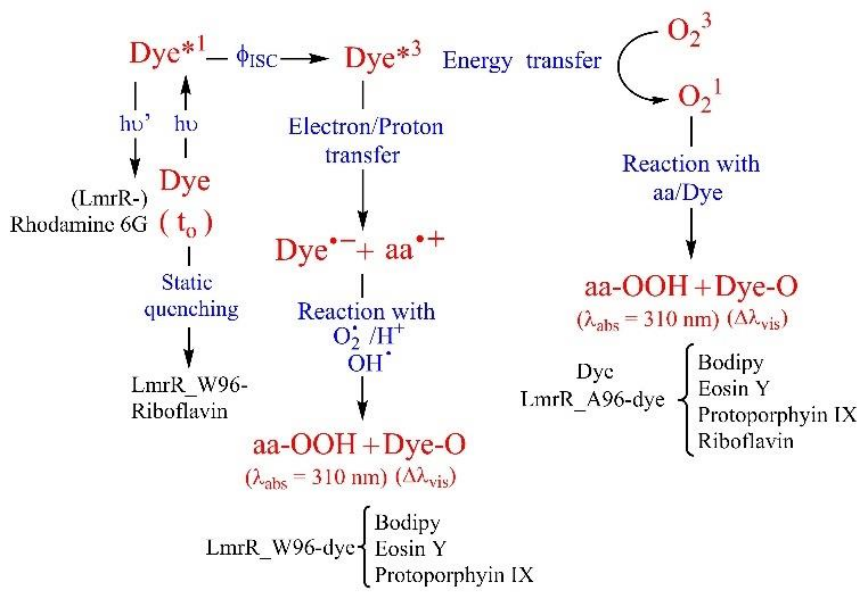

Figure 8. Potential pathways for dye photodegradation and amino acid oxidation induced by dye photoreactions. The triplet state of the dye $\left(\mathrm{Dye}^{3}\right)$ can either form singlet oxygen $\left(\mathrm{O}_{2}\right)$ by energy transfer or abstract an electron rom susceptible amino acids producing amino acid radical (AA - and dye anion radical (Dye-) Amino acid and dye radicals can react with oxygen radicals $\left(\mathrm{O}_{2}\right)$ or hydroxy Dye-O and AA-OOH. Singlet oxygen can oxidize neutral amino acids or dyes directly also (right pathway).

\section{Conclusions}

LmrR is a transcription factor that controls the expression of the heterodimeric $A B C$ transporter $L m r C D$, which is a major multidrug transporter in Lactococcus lactis bacteria. ${ }^{1415} \mathrm{LmrCD}$ transporters act as efflux pumps for antibiotics which is one of the mechanism for bacterial drug resistance. Recently, it has been shown that some toxic compounds such as the DNAbinding drugs Hoechst 33342 (H33342) interact directly with LmrR and its presence in the growth medium induces a significant upregulation of the $\operatorname{ImrCD}$ genes and higher resistance to antibiotics. ${ }^{83}$

The present study shows that LmrR, and especially the tryptophan residue, has a major impact on the photochemistry and photobleaching of dyes. The variation in behaviour observed in the present study indicates that the behaviour of dyes in general cannot be easily assumed based on their properties in aqueous or organic solvents. The rates of photodegradation observed here, although relative, may be general to other protein systems as the photostability and photochemistry of dyes embedded in LmrR strongly depends on protein-dye interactions and the relative susceptibility of the protein itself to react with ${ }^{1} \mathrm{O}_{2}$ among others. Finally, the complex interaction of ${ }^{1} \mathrm{O}_{2}$ with the protein observed in this study holds implications for our expectations of how photochemically active dyes will behave when moving from solution studies to in vivo applications. In the latter case, protein dye interactions need to be considered in the dyes overall activity, not least in PDT antibacterial applications. The efficiency of the photodynamic therapy relies on the generation of ROS as superoxide anions, hydrogen peroxide $\left(\mathrm{H}_{2} \mathrm{O}_{2}\right)$, free hydroxyl radicals $\left(\mathrm{OH}^{\circ}\right)$, and singlet oxygen $\left({ }^{1} \mathrm{O}_{2}\right)$ by the drugs inside the cell. ${ }^{84}$ These ROS oxidize various biomolecules causing damage in the cell. In spite of the multitarget approach of photodynamic therapy, bacteria can developed defence to 
oxidative stress generated by ROS by specific enzymatic pathways. ${ }^{85-87}$ However, it is not clear how bacterial mechanisms deal with singlet oxygen. ${ }^{84}$ Our work shows a potentially important pathway in bacterial resistance to singlet oxygen by preventing its formation in the first place.

\section{Methods}

Gene construction and cloning. The gene encoding for LmrR is based on that used in our previously reported PET17b_LmrR_LM. ${ }^{39}$ The LmrR gene includes the K55D and K59Q modifications and a modified C-terminal strep-tag (tag amino acid sequence is ASHPQFEL). LmrR_W96 was generated by introducing W67A and W124A modifications on the LmrR gene and LmrR_W96A includes also the W96A modification. The modification on the plasmids were carried out by quickchange site-directed mutagenesis protocol.

Expression and purification. The expression of the different protein variants was performed as described previously with minor modifications. ${ }^{39}$ Briefly, protein expression plasmids of the $L m r R$ constructs were transformed into $E$. coli BL21 (DE3)_C43. Cells were grown in LB media containing $0.1 \mathrm{mg} / \mathrm{mL}$ ampicillin at $37^{\circ} \mathrm{C}$ to an OD at $600 \mathrm{~nm}$ of $0.6-0.8$. The expression was induced with $1 \mathrm{mM}$ IPTG and the cells were grown overnight at $30^{\circ} \mathrm{C}$.

The proteins were expressed as strep-tagged proteins and purified using standard affinity chromatography methods. Briefly, cells were harvested by centrifugation during $15 \mathrm{~min}$ at $4700 \mathrm{~g}$ and resuspended in buffer $\left(50 \mathrm{mM} \mathrm{NaH}_{2} \mathrm{PO}_{4}, \mathrm{pH} 8.0,150\right.$ $\mathrm{mM} \mathrm{NaCl})$. Cells were lysed by sonication (75\% (200W) for $8 \mathrm{~min}$ (10 s on, $15 \mathrm{~s}$ off)) and the lysed cells were incubated with DNAsel $(0.1 \mathrm{mg} / \mathrm{mL}$, containing final concentration $10 \mathrm{mM}$ $\mathrm{MgCl}_{2}$ ) for 1 hour at $4^{\circ} \mathrm{C}$.

After centrifugation (15000 rpm, JA-17, $1 \mathrm{~h}, 4{ }^{\circ} \mathrm{C}$, Beckman), the supernatant was equilibrated with $6 \mathrm{~mL}$ of pre-equilibrated Strep-tag Tactin column material for $1 \mathrm{~h}$ (mixed at $200 \mathrm{rpm}$ on a rotary shaker) at room temperature. The column was washed with $3 \times 1 \mathrm{CV}$ (column volume) of resuspension buffer (same buffer as used before), and eluted with $6 \times 0.5 \mathrm{CV}$ of resuspension buffer containing $2.5 \mathrm{mM}$ desthiobiotin. The impurities were removed from pure LmrR by FPLC using a Superdex 200 size exclusion column (GE Healthcare) in $50 \mathrm{mM}$ potassium phosphate $\mathrm{pH} 8$ buffer. The protein size and purity were analyzed by SDS polyacrylamide gel electrophoresis. The concentration of LmrR_W96 is determined by using the calculated molar absorptivities, $\varepsilon_{280}=28880 \mathrm{M}^{-1} \mathrm{~cm}^{-1}$, and LmrR_W96A $\varepsilon_{280}=17880 \mathrm{M}^{-1} \mathrm{~cm}^{-1}$ (calculations were done by Protparam on the Expasy server. http://web.expasy.org/protpara). Purified proteins were stored in dark frozen at $-80^{\circ} \mathrm{C}$.

Protein-dye binding determination. UV.Vis absorption spectra were recorded using a SPECORD 5600 UV-vis spectrophotometer. Binding was monitored by absorbance peak shift (specified in the figures to each dye) at room temperature in a $1 \mathrm{~cm}$ pathlength quartz cuvette. Stock solutions of dyes were prepared $(800 \mu \mathrm{M})$, in $50 \mathrm{mM}$ phosphate buffer $\mathrm{pH} 8$ in the case of rose bengal and rhodamine 6G, in methanol in the case of protoporphyin IX and bodipy, in ethanol in the case of eosin $\mathrm{Y}$ and in $10 \%$ of acetonitrile in the case of riboflavin. $2 \mathrm{ml}$ at $6 \mu \mathrm{M}$ of rose bengal, $10 \mu \mathrm{M}$ of protoporphyin IX, $10 \mu \mathrm{M}$ of bodipy, $30 \mu \mathrm{M}$ of riboflavin, $4 \mu \mathrm{M}$ of eosin $\mathrm{Y}$ and $6 \mu \mathrm{M}$ rhodamine $6 \mathrm{G}$ on $50 \mathrm{mM}$ phosphate buffer $\mathrm{pH} 8$ was used as starting point. Titration of protein to dye were carried out in the range of 0.1 to 4 equiv. initial dye concentration.

Circular dichroism (CD) spectroscopy. CD spectra were recorded using a JASCO J-810 CD Spectrometer. CD spectra of LmrR protein in $50 \mathrm{mM}$ phosphate buffer at $\mathrm{pH} 8$ were acquired in a $1 \mathrm{~cm}$ path length quartz cuvettes at a protein concentration of $1 \mu \mathrm{M}$ and with or without $10 \mu \mathrm{M}$ dye. A band-width of $1 \mathrm{~nm}$ and $1 \mathrm{~nm}$ increment were used in all cases. Thermal denaturation was followed by monitoring the ellipticity at $222 \mathrm{~nm}$ at $5^{\circ} \mathrm{C}$ intervals. Emission spectroscopy. Emission spectra were recorded using a JASCO_FP6200 spectrophotomer at room temperature in a 1 $\mathrm{cm}$ pathlength quartz cuvette and using a $310 \mathrm{~nm}$ long-pass filter at the detector side. $2 \mathrm{ml}$ at $2 \mu \mathrm{M}$ of rose bengal, $5 \mu \mathrm{M}$ of protoporphyrin IX, $0.25 \mu \mathrm{M}$ of bodipy, $0.5 \mu \mathrm{M}$ of riboflavin, $0.1 \mu \mathrm{M}$ of eosin $\mathrm{Y}$ and $0.1 \mu \mathrm{M}$ rhodamine $6 \mathrm{G}$ in $50 \mathrm{mM}$ phosphate buffer $\mathrm{pH}$ 8 was measured in the absence of protein and in the presence of a four-fold excess of LmrR_W96 and LmrR_W96A. Emission spectra were corrected for instrument response and absorbance.

Quenching of Tryptophan fluorescence. Dye (as a stock solution) was added to $1 \mathrm{~mL}$ of a buffered solution (50 mM phosphate buffer, pH 8) containing $1 \mu \mathrm{M}$ LmrR_W96 in a step wise manner from 0.1 to 10 equiv. with respect to the initial protein concentration in $1 \mathrm{~cm}$ pathlength cuvettes. Emission spectra were recorded after 3-5 min incubation time at room temperature with excitation at $280 \mathrm{~nm}$ unless stated otherwise. The spectra were corrected for detector response and absorbance. The integrated emission from 300-420 nm was plotted against the concentration of the dye.

Emission lifetimes. A PicoQuant PDL 800-B diode laser driver connected to a PicoQuant PLS 255 led-head equipped with 250$350 \mathrm{~nm}$ bandpass filter provided excitation light. TCSPC was carried out using a PicoQuant Tau-SPAD-100 single photon counting module equipped with 325-385 nm bandpass filter and connected to a PicoQuant PicoHarp 300 TC-SPC module. The Tau SPAD was powered by a DSN 102 dual SPAD power supply. The internal trigger from the PDL was used as input for time stamping on the PicoHarp and the emitted light was measured perpendicular to the excitation light. Lifetimes were calculated using FluoFit with correction for the IRF, obtained by scattering from $\mathrm{BaSO}_{4}$ at $45^{\circ}$ relative to the excitation light.

Singlet oxygen measurements. ${ }^{1} \mathrm{O}_{2}$ emission generated upon excitation at $455 \mathrm{~nm}(50 \mathrm{~mW})$ was detected $1 \mathrm{~cm}$ pathlength quartz cuvettes using a cuvette holder with collimator for collection of the emitted light (Thorlabs) equipped with a 950 $\mathrm{nm}$ long pass filter and delivery by fiber optic to an Andor Technology Shamrock300i spectrograph equipped with a 75 I/mm (1300 nm blazed) grating and idus-InGaAs 512 pixel diode array (Andor Technology). Spectral calibration was performed with the emission lines from a TL light. $30 \mu \mathrm{M}$ of riboflavin or $\left[R u(b p y)_{3}\right]^{2+}$ in deuterated $50 \mathrm{mM}$ phosphate buffer $\mathrm{pD} 8$ was 
measure in the absence and presence of $120 \mu \mathrm{M}$ LmrR_W96 and LmrR_W96A protein.

Photochemical stability. UV/vis absorption spectra were typically recorded at $30 \mathrm{~s}$ intervals during continuous irradiation at room temperature in $1 \mathrm{~cm}$ pathlength quartz cuvettes. 800 $\mu \mathrm{M}$ dye stock solutions were prepared, prior to experiments, in 50 $\mathrm{mM}$ phosphate buffer in $\mathrm{D}_{2} \mathrm{O}$ pD 8 in the case of rose bengal and rhodamine $6 \mathrm{G}$, in methanol in the case of protoporphyin IX and bodipy, in ethanol in the case of eosin $\mathrm{Y}$ and in $10 \%$ of acetonitrile in water in the case of riboflavin. From these solutions $2 \mathrm{ml}$ of $6 \mu \mathrm{M}$ rose bengal, $10 \mu \mathrm{M}$ protoporphyin IX, $10 \mu \mathrm{M}$ bodipy, $30 \mu \mathrm{M}$ riboflavin , $4 \mu \mathrm{M}$ eosin $\mathrm{Y}$, and $6 \mu \mathrm{M}$ rhodamine $6 \mathrm{G}$ in $50 \mathrm{mM}$ phosphate buffer in $\mathrm{D}_{2} \mathrm{O}$ at $\mathrm{pD} 8$ were used both with and without a 4 fold excess of LmrR_W96 or LmrR_W96A. The proteins were prepared by lyophilisation followed by dissolution in $\mathrm{D}_{2} \mathrm{O}$. Solutions were irradiated with at 505 or $\mathbf{4 5 5} \mathrm{nm}$ using the collimated output of high power LEDs (Thorlabs) such that the entire output passed through the cuvette as a $7 \mathrm{~mm}$ beam. Oxygen was removed from the sample by purging with argon for $15 \mathrm{~min}$.

Calculation of excited state redox potential calculation. The equations for Gibbs free energy of photoinduced electron transfer (equation 8 and 9) (69,88 $^{6}$ were used to calculated excited state redox potentials. Ground state reduction and oxidation potentials were obtained from literature values. ${ }^{89-94}$ Electrostatic work for charge generation and separation is approximated to 0 and energy gap between zeroth vibrational levels of the ground and excited state $\left(E_{o o}\right)$ is approximated as the intercept of the normalized emission and absorption spectra of the LmrR_W96-dye complex.

$\mathrm{E}_{1 / 2} *$ red $=\mathrm{E}_{1 / 2}{ }^{\mathrm{red}}+\mathrm{E}_{\mathrm{oo}}+\mathrm{w}_{\mathrm{r}}$

(equation 8)

$\mathrm{E}_{1 / 2} * \mathrm{ox}=\mathrm{E}_{1 / 2}{ }^{\mathrm{ox}}-\mathrm{E}_{\mathrm{oo}}+\mathrm{w}_{\mathrm{r}}$

(equation 9)

\section{Conflicts of interest}

There are no conflicts to declare.

\section{Acknowledgements}

Financial support was provided by The Netherlands Ministry of Education, Culture and Science (Gravity Program 024.001.035 to G.R. and W.R.B.).

\section{References}

R. W. Redmond and J. N. Gamlin, Photochem. Photobiol., 1999, 70, 391-475.

2 G. Stark, J. Membr. Biol., 2005, 205, 1-16.

3 J. L. Ravanat, P. Di Mascio, G. R. Martinez, M. H. G. Medeiros and J. Cadet, J. Biol. Chem., 2001, 276, 4060140604.

4 V. Vargová, R. E. Giménez, H. Černocká, D. C. Trujillo, F. Tulli, V. I. P. Zanini, E. Paleček, C. D. Borsarelli and V. Ostatná, Electrochim. Acta, 2016, 187, 662-669.

5 T. Dai, Y. Y. Huang and M. R. Hamblin, Photodiagnosis Photodyn. Ther., 2009, 6, 170-188.

6 X. Ragàs, D. Sánchez-García, R. Ruiz-González, T. Dai, M.
Agut, M. R. Hamblin and S. Nonell, J. Med. Chem., 2010, 53, 7796-7803.

S. A. G. Lambrechts, T. N. Demidova, M. C. G. Aalders, T. Hasan and M. R. Hamblin, Photochem. Photobiol. Sci., 2005, 4, 503-9.

T. Maisch, Mini-Reviews Med. Chem., 2009, 9, 974-983. H. B. Gray and J. R. Winkler, Proc. Natl. Acad. Sci., 2015, 112, 10920-10925.

10 C. F. Higgins, Nature, 2007, 446, 749-757.

11 P. K. Madoori, H. Agustiandari, A. J. M. Driessen and A. M. W. H. Thunnissen, EMBO J., 2009, 28, 156-166.

12 H. Agustiandari, J. Lubelski, H. B. Van Den Berg Van Saparoea, O. P. Kuipers and A. J. M. Driessen, J. Bacteriol., 2008, 190, 759-763.

H. Agustiandari, E. Peeters, J. G. de Wit, D. Charlier and A. J. M. Driessen, Microbiology, 2011, 157, 1519-1530.

H. Agustiandari, J. Lubelski, H. B. Van Den Berg Van Saparoea, O. P. Kuipers and A. J. M. Driessen, J. Bacteriol., 2008, 190, 759-763.

H. Agustiandari, E. Peeters, J. G. de Wit, D. Charlier and A. J. M. Driessen, Microbiology, 2011, 157, 1519-1530. K. Takeuchi, Y. Tokunaga, M. Imai, H. Takahashi and I. Shimada, Sci. Rep., 2014, 4, 6922.

J. P. Van Der Berg, P. K. Madoori, A. G. Komarudin, A. M. Thunnissen, A. J. M. Driessen and S. Korolev, PLoS One, , DOI:10.1371/journal.pone.0135467. H. Wade, Curr. Opin. Struct. Biol., 2010, 20, 489-496. K. Takeuchi, Y. Tokunaga, M. Imai, H. Takahashi and I. Shimada, Sci. Rep., 2015, 4, 6922.

R. Schweitzer-Stenner, J. Porphyr. Phthalocyanines, 2011, 15, 312-337.

E. S. Manas, J. M. Vanderkooi and K. A. Sharp, J. Phys. Chem. B, 1999, 103, 6334-6348.

H. Khoa Ly, M. Sezer, N. Wisitruangsakul, J. J. Feng, A. Kranich, D. Millo, I. M. Weidinger, I. Zebger, D. H. Murgida and P. Hildebrandt, FEBS J., 2011, 278, 1382-1390. 66.

J. E. Hill, M. K. Linder, K. S. Davies, G. A. Sawada, J. Morgan, T. Y. Ohulchanskyy and M. R. Detty, J. Med. Chem., 2014, 57, 8622-8634.

Z. Fan, X. Cui, D. Wei, W. Liu, B. Li, H. He, H. Ye, N. Zhu and X. Wei, Sci. Rep., 2016, 6, 25353.

E. Blake, J. Allen and A. Curnow, Photodiagnosis Photodyn. Ther., 2013, 10, 575-582.

K. S. Davies, M. K. Linder, M. W. Kryman and M. R. Detty, Bioorganic Med. Chem., 2016, 24, 3908-3917.

28 J. P. M. L. Rolim, M. A. S. De-Melo, S. F. Guedes, F. B. Albuquerque-Filho, J. R. De Souza, N. A. P. Nogueira, I. C. J. Zanin and L. K. A. Rodrigues, J. Photochem. Photobiol. B Biol., 2012, 106, 40-46.

S. T. G. Buck, F. Bettanin, E. Orestes, P. Homem-de-Mello, H. Imasato, R. B. Viana, J. R. Perussi and A. B. F. da Silva, J. Chem., 2017, 2017, 1-9.

A. Arboleda, D. Miller, F. Cabot, M. Taneja, M. C. Aguilar, K. Alawa, G. Amescua, S. H. Yoo and J.-M. Parel, Am. J. Ophthalmol., 2014, 158, 64-70. 
Mol. Biomol. Spectrosc., 2011, 81, 458-465.

M. A. Rauf, J. P. Graham, S. B. Bukallah and M. A. S. Al-

Saedi, Spectrochim. Acta - Part A Mol. Biomol. Spectrosc., 2009, 72, 133-137. Phys. Chem. Chem. Phys., 2016, 18, 16266-16273.

F. M. Zehentbauer, C. Moretto, R. Stephen, T. Thevar, J. R. Gilchrist, D. Pokrajac, K. L. Richard and J. Kiefer, Spectrochim. Acta - Part A Mol. Biomol. Spectrosc., 2014, 121, 147-151.

S. Terdale and A. Tantray, J. Mol. Liq., 2017, 225, 662-671.

G. R. Brunk, K. A. Martin and A. M. Nishimura, Biophys. J., 1976, 16, 1373-1384.

N. M. B. Neto, D. S. Correa, L. De Boni, G. G. Parra, L. Misoguti, C. R. Mendonça, I. E. Borissevitch, S. C. Zílio and P. J. Gonçalves, Chem. Phys. Lett., 2013, 587, 118-123. J. Bos, F. Fusetti, A. J. M. Driessen and G. Roelfes, Angew. Chemie Int. Ed., 2012, 51, 7472-7475.

J. Bos, W. R. Browne, A. J. M. Driessen and G. Roelfes, J. Am. Chem. Soc., 2015, 137, 9796-9799.

T. A. Schüttrigkeit, C. K. Kompa, M. Salomon, W. Rüdiger
L. Villarino, K. E. Splan, E. Reddem, L. Alonso-Cotchico, C. Gutiérrez de Souza, A. Lledós, J.-D. Maréchal, A.-M. W. H. Thunnissen and G. Roelfes, Angew. Chemie Int. Ed., 2018, 57, 7785-7789.

R. G. Huber, M. A. Margreiter, J. E. Fuchs, S. Von Grafenstein, C. S. Tautermann, K. R. Liedl and T. Fox, J. Chem. Inf. Model., 2014, 54, 1371-1379.

R. G. Huber, M. A. Margreiter, J. E. Fuchs, S. Von Grafenstein, C. S. Tautermann, K. R. Liedl and T. Fox, J. Chem. Inf. Model., 2014, 54, 1371-1379.

C. R. Martinez and B. L. Iverson, Chem. Sci., 2012, 3, 2191. Y. Deng, X. Feng, D. Yang, C. Yi and X. Qiu, BioResources, 2012, 7, 1145-1156.

L. Li, B. Nguyen and K. Burgess, Bioorganic Med. Chem. Lett., 2008, 18, 3112-3116.

S. Al-Ani, ISESCO J. Sci. Technol., 2013, Vo I ume 9, ( 3 4-4 2 ).

X. Shu, V. Lev-Ram, T. J. Deerinck, Y. Qi, E. B. Ramko, M. W. Davidson, Y. Jin, M. H. Ellisman and R. Y. Tsien, PLoS Biol., 2011, 9, e1001041. and M. E. Michel-Beyerle, Chem. Phys., 2003, 294, 501508.

66

67 Nonlinear Sci., 2009, 7, 290-300.

E. Alarcón, A. M. Edwards, A. Aspée, C. D. Borsarelli and E. a Lissi, Photochem. Photobiol. Sci., 2009, 8, 933-43.

M. B. E. Turbay, V. Rey, N. M. Argañaraz, F. E. M. Vieyra, A Aspée, E. A. Lissi and C. D. Borsarelli, J. Photochem. Photobiol. B Biol., 2014, 141, 275-282.

N. C. Maiti, S. Mazumdar and N. Periasamy, J. Phys. Chem. B, 1998, 102, 1528-1538.

F. Würthner, T. E. Kaiser and C. R. Saha-Möller, Angew. Chemie - Int. Ed., 2011, 50, 3376-3410.

J.-D. Wang, S. Li, R. Lü and A. Yu, Wuli Huaxue Xuebao/ Acta Phys. - Chim. Sin., 2015, 31, 1787-1794.

N. Dorh, S. Zhu, K. B. Dhungana, R. Pati, F.-T. Luo, H. Liu and A. Tiwari, Sci. Rep., 2015, 5, 18337.

G. N. Lewis and M. Calvin, Chem. Rev., 1939, 25, 273-328.

B. Hinkeldey, A. Schmitt and G. Jung, ChemPhysChem,

2008, 9, 2019-2027.

L. Shen and H. F. Ji, J. Photochem. Photobiol. B Biol., 2008, 92, 10-12.

P. Li, S. Liu, X. Wang, Z. Liu and Y. He, Luminescence, 2013, 28, 910-914.

H. Staudt, D. Oesterhelt, M. Grininger and J. Wachtveitl, J. Biol. Chem., 2012, 287, 17637-17644.

M. J. Davies, Biochem. J., 2016, 473, 805-825.

F. M. Pimenta, R. L. Jensen, T. Breitenbach, M. Etzerodt and P. R. Ogilby, Photochem. Photobiol., 2013, 89, 11161126.

J. M. Gebicki, Arch. Biochem. Biophys., 2016, 595, 33-39.

R. Ruiz-González, A. L. Cortajarena, S. H. Mejias, M. Agut, S. Nonell and C. Flors, J. Am. Chem. Soc., 2013, 135, 95649567.

C. TAnielian and G. HEINRICH, Photochem. Photobiol., 1995, 61, 131-135.

R. F. Khairutdinov and N. Serpone, J. Phys. Chem., 1995, 99, 11952-11958.

S. A. Soper, H. L. Nutter, R. A. Keller, L. M. Davis and E. B. Shera, Photochem. Photobiol., 1993, 57, 972-977.

J. Widengren and R. Rigler, Bioimaging, 1996, 4, 149-157. D. Rehm and A. Weller, Isr. J. Chem., 1970, 8, 259-271. S. D. Glover, R. Tyburski, L. Liang, C. Tommos and L. Hammarström, J. Am. Chem. Soc., 2018, 140, 185-192. P. Walrant and R. Santus, Photochem. Photobiol., 1974, 19, 411-417.

Y. Fukunaga, Y. Katsuragi, T. Izumi and F. Sakiyama, J. Biochem., 1982, 92, 129-141.

M. S. Hamdy, E. L. Scott, R. H. Carr and J. P. M. Sanders, Catal. Letters, 2012, 142, 338-344.

M. Różanowska, J. Ciszewska, W. Korytowski and T. Sarna, J. Photochem. Photobiol. B Biol., 1995, 29, 71-77.

M. Geoffroy, P. Lambelet and P. Richert, J. Agric. Food Chem., 2000, 48, 974-978.

M. Gouterman, J. Mol. Spectrosc., 1961, 6, 138-163. I. Ahmad and F. H. M. Vaid, Compr. Ser. Photochem. Photobiol. Sci., 2006, 6, 13-40.

P. F. Heelis, Chem. Soc. Rev., 1982, 11, 15.

S. Astanov, M. Z. Sharipov, A. R. Fayzullaev, E. N. Kurtaliev and N. Nizomov, J. Mol. Struct., 2014, 1071, 133-138. M. A. Sheraz, S. H. Kazi, S. Ahmed, Z. Anwar and I. Ahmad, Beilstein J. Org. Chem., 2014, 10, 1999-2012.

81 E. Silva, P. Barrias, E. Fuentes-Lemus, C. Tirapegui, A. Aspee, L. Carroll, M. J. Davies and C. López-Alarcón, Free Radic. Biol. Med., 2019, 131, 133-143. K. Hirakawa and H. Ito, Chem. Phys. Lett., 2015, 627, 2629.

P. K. Madoori, H. Agustiandari, A. J. M. Driessen and A. M W. H. Thunnissen, EMBO J., 2009, 28, 156-166.

F. Cieplik, D. Deng, W. Crielaard, W. Buchalla, E. Hellwig, A. Al-Ahmad and T. Maisch, Crit. Rev. Microbiol., 2018, 571589. 
Gwizdek-Wiśniewska and K. P. Bielawski, BMC Microbiol., 2010, 10, 323

86 M. Tim, J. Photochem. Photobiol. B Biol., 2015, 150, 2-10.

87 N. Kashef and M. R. Hamblin, Drug Resist. Updat., 2017, 31, 31-42.

88 G. J. Kavarnos, Fundamentals of photoinduced electron transfer, VCH Publishers, 1993.

89 K. Fidaly, C. Ceballos, A. Falguières, M. S. I. Veitia, A. Guy and C. Ferroud, Green Chem., 2012, 14, 1293-1297.

90 V. Srivastava and P. P. Singh, RSC Adv., 2017, 7, 3137731392.

91 C. L. Hou, Y. Yao, D. Wang, J. Zhang and J. L. Zhang, Org. Chem. Front., 2019, 6, 2266-2274.

92 K. M. Schaich, Lipids, 1992, 27, 209-218.

93 S. L. J. Tan and R. D. Webster, J. Am. Chem. Soc., 2012, 134, 5954-5964.

94 S. Doose, H. Neuweiler and M. Sauer, ChemPhysChem, 2009, 10, 1389-1398. 


\section{Electronic Supporting information}

Impact of binding to the Multidrug Resistant Regulator protein LmrR on the photophysics and -chemistry of photosensitizers

Sara H. Mejías, ${ }^{*, a}$ Gerard Roelfes, ${ }^{a}$ and Wesley R. Browne*,a

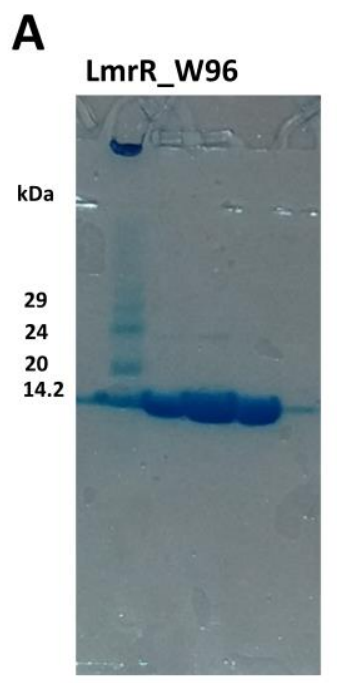

B

LmrR_A96

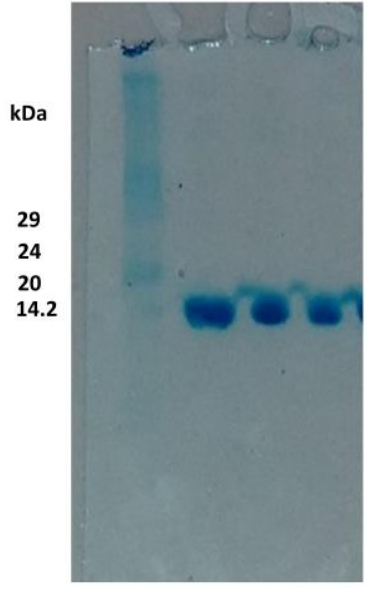

Figure S1. SDS-page gels of purified LmrR mutants. Left, LmrR_W96, and right, LmrR_A96.
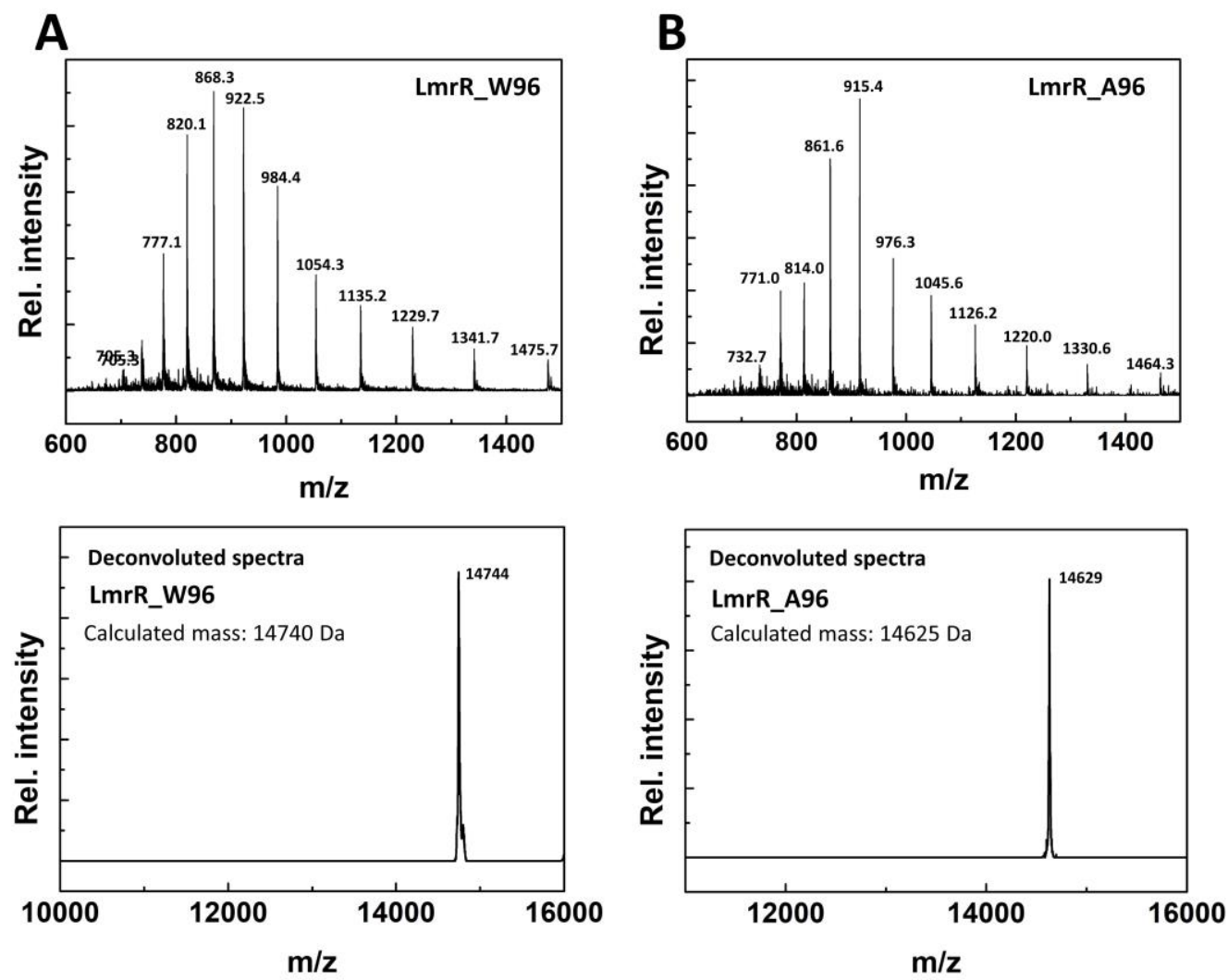

Figure S2. Electrospray ionization (ESI) mass spectra LmrR_W96 and LmrR_A96. 
A

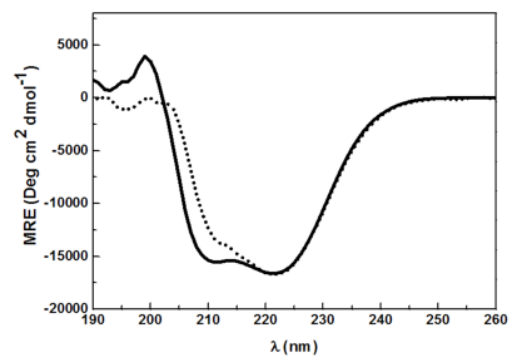

B

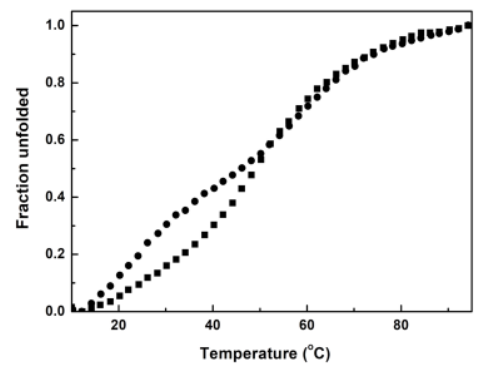

C

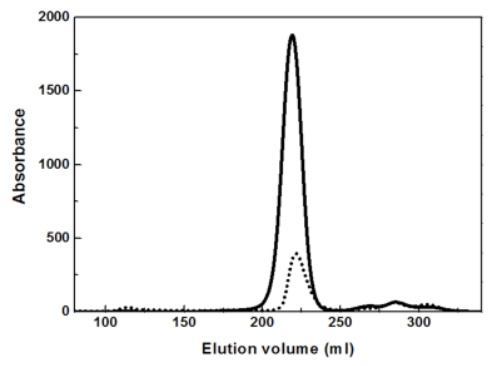

Figure S3. (a) Circular dichroism spectrum, (b) thermal denaturation and (c) size exclusion chromatography of LmrR_W96 (solid lines and squares) and LmrR_W96A (dotted lines).
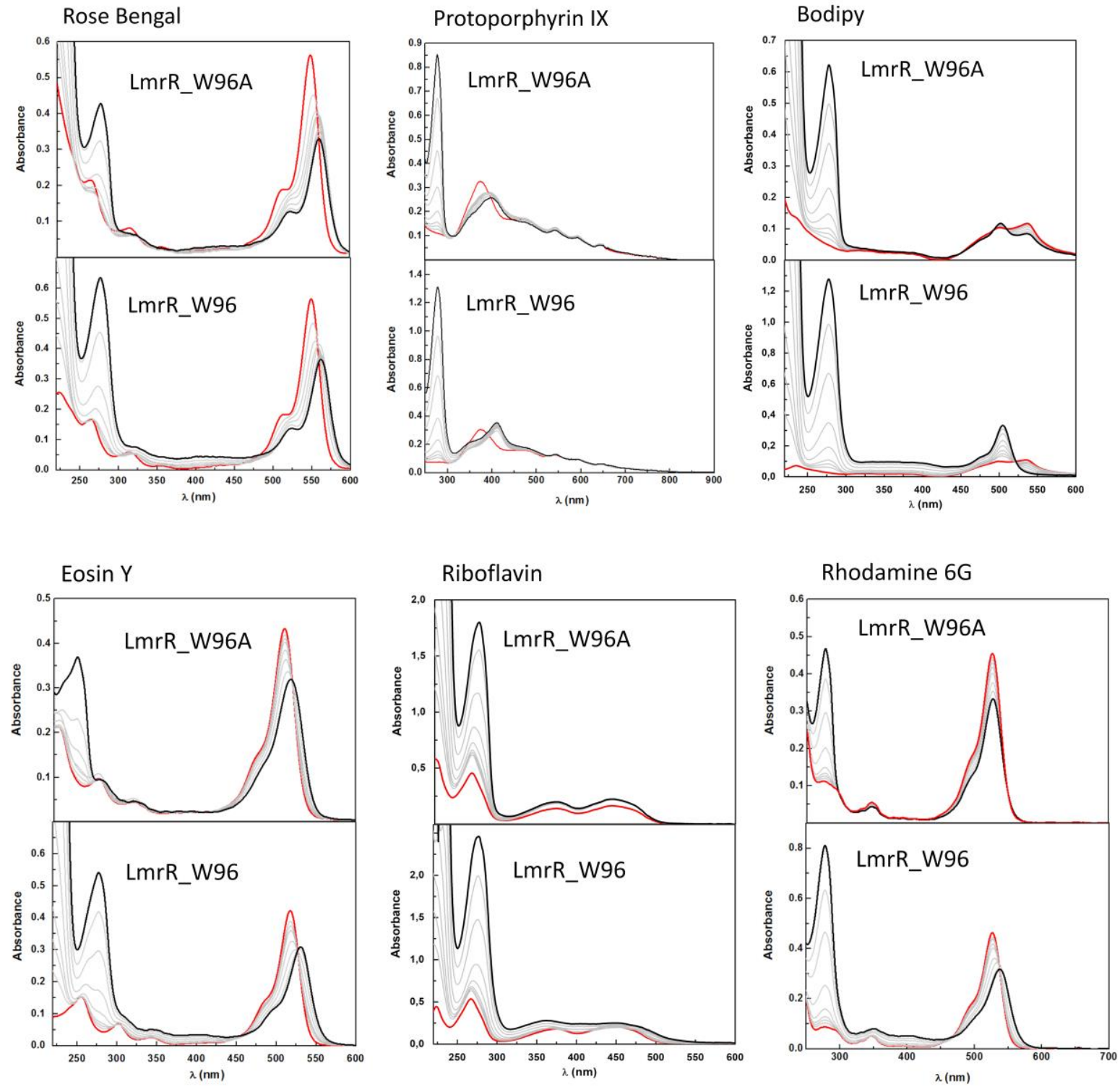

Figure S4. UV-vis absorbance spectra of dyes (at constant concentration) with increasing concentration from 0.1 equiv. (red line) to 10 equiv. (black line) of LmrR_W96A or LmrR_W96. 
A

$$
\begin{aligned}
& \text { - LmrR_W96 } \\
& \text { - LmrR_W96+Fluorophore }
\end{aligned}
$$

\section{LmrR_W96 + RB}

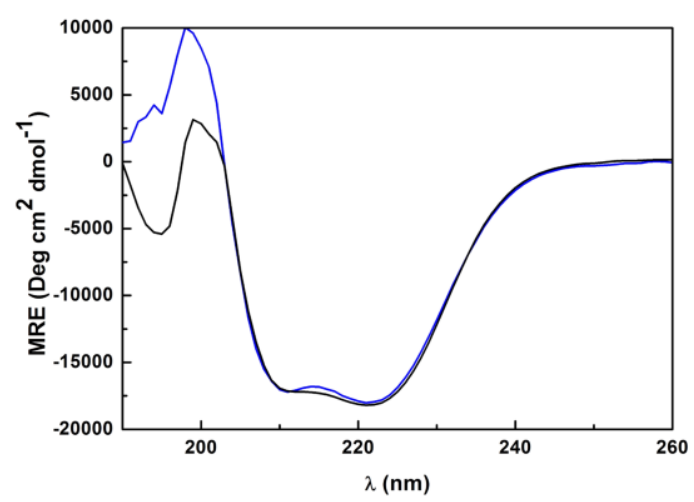

\section{LmrR_W96 + PpIX}

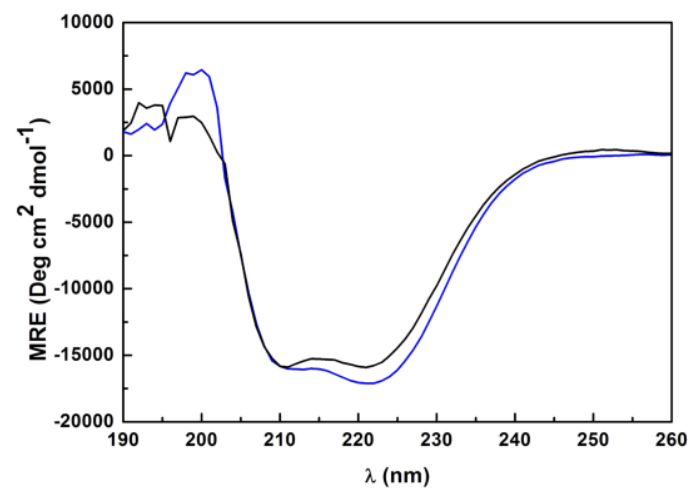

\section{LmrR_W96 + Bodipy}

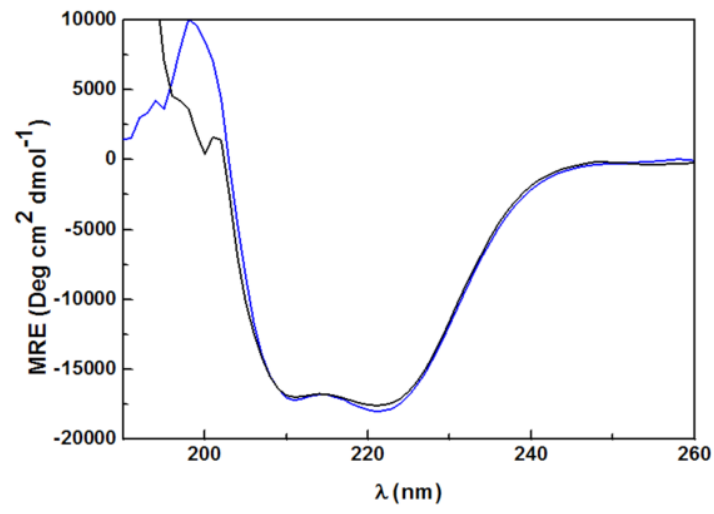

B
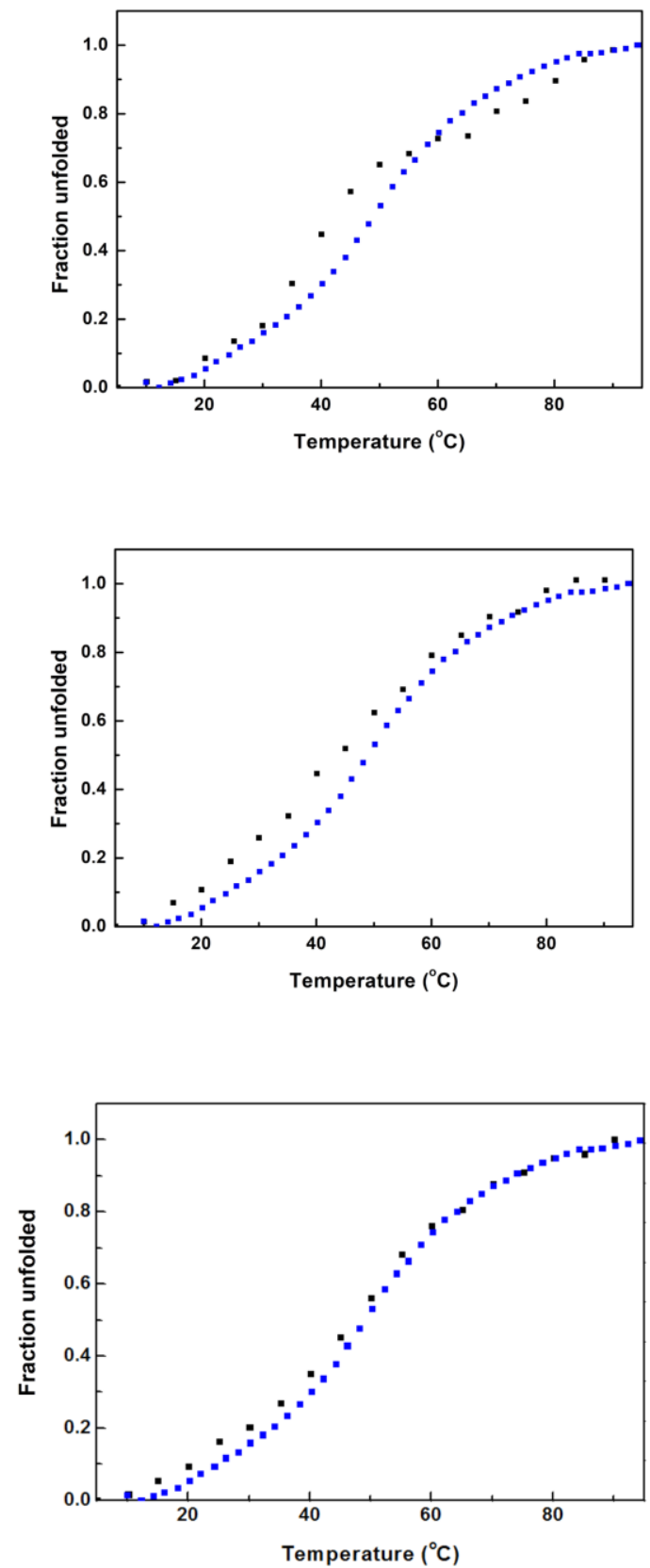
LmrR_W96 + EY

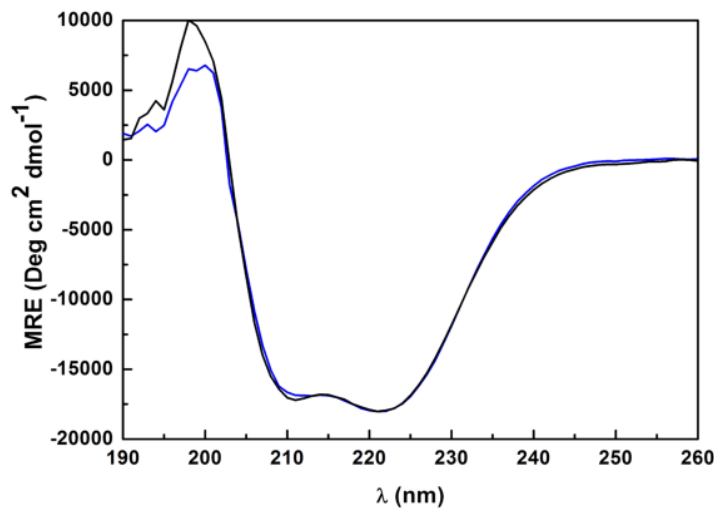

LmrR_W96 + RBF

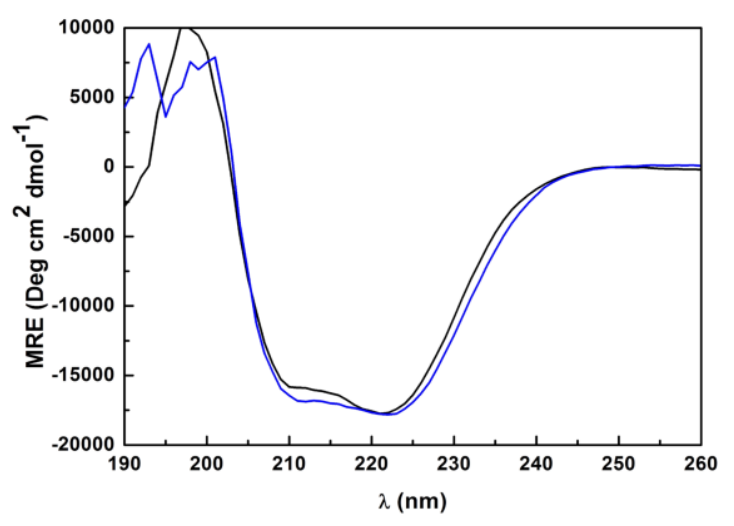

LmrR_W96 + Rh6G

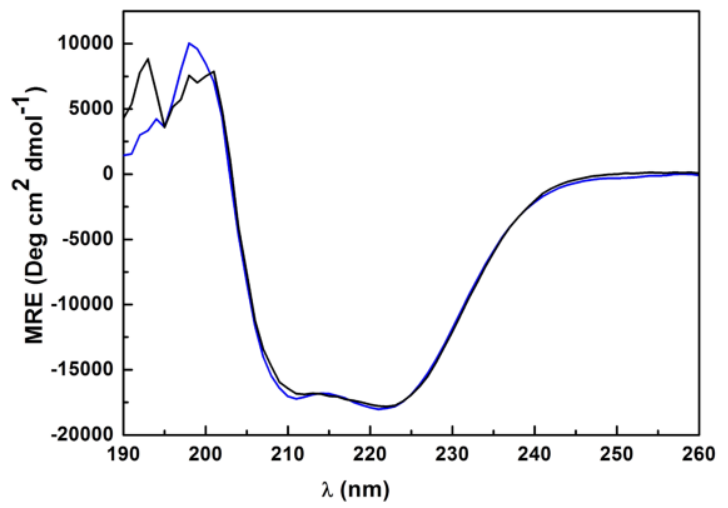

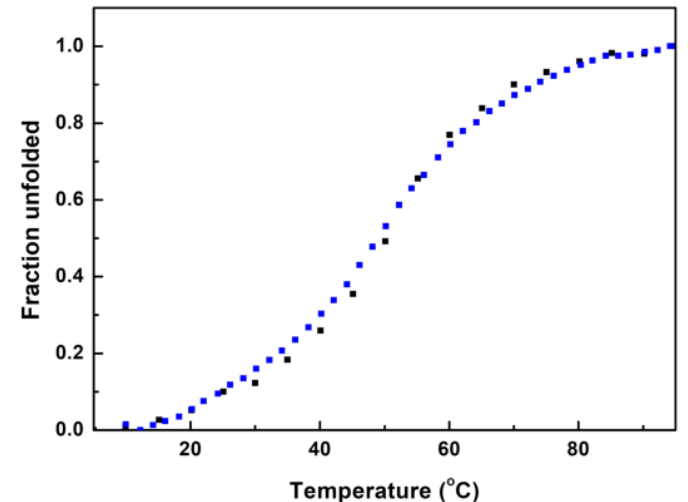
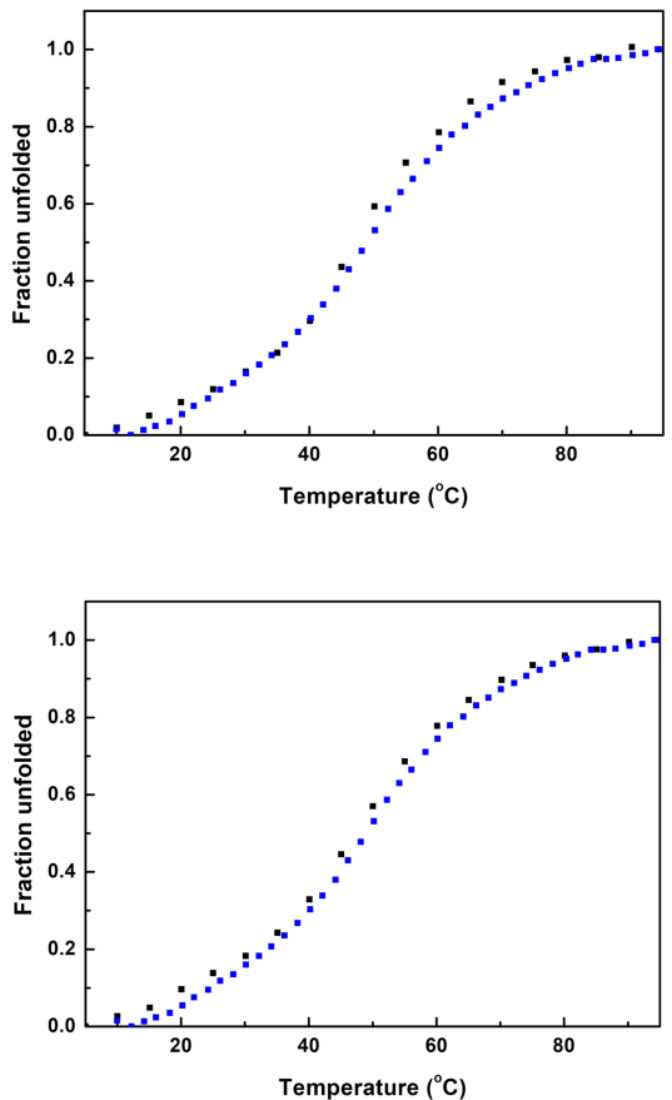

Figure S5. (a) Circular dichroism spectra and (b) thermal denaturation of LmrR_W96 (blue) and LmrR_W96 in the presence of the dyes (black). 

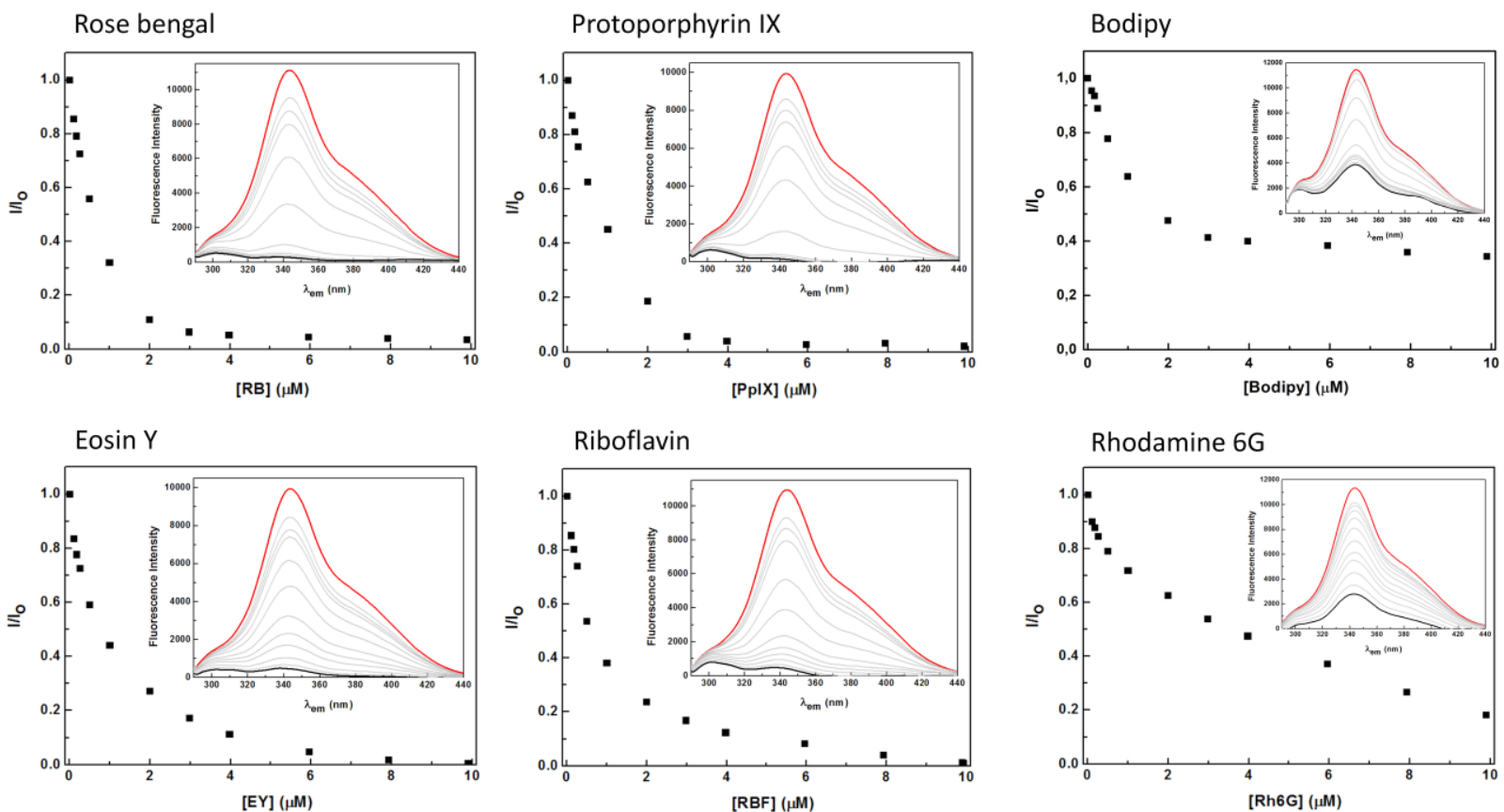

Figure S6. Tryptophan emission in the presence of dye (I) divided by the intensity of tryptophan emission in the absence of the dye $\left(I_{0}\right)$. Insets, tryptophan fluorescence $\left(\lambda_{\text {ex }} 280 \mathrm{~nm}\right)$ of $1 \mu \mathrm{M}$ LmrR_W96 at increasing dye concentration from 0 , red line, to $10 \mu \mathrm{M}$, black line. 
IRF (Instrument response function)

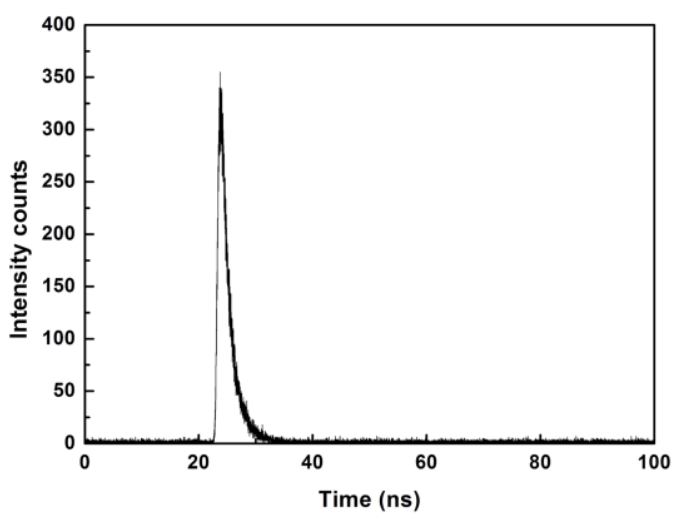

LmrR_W96+RB
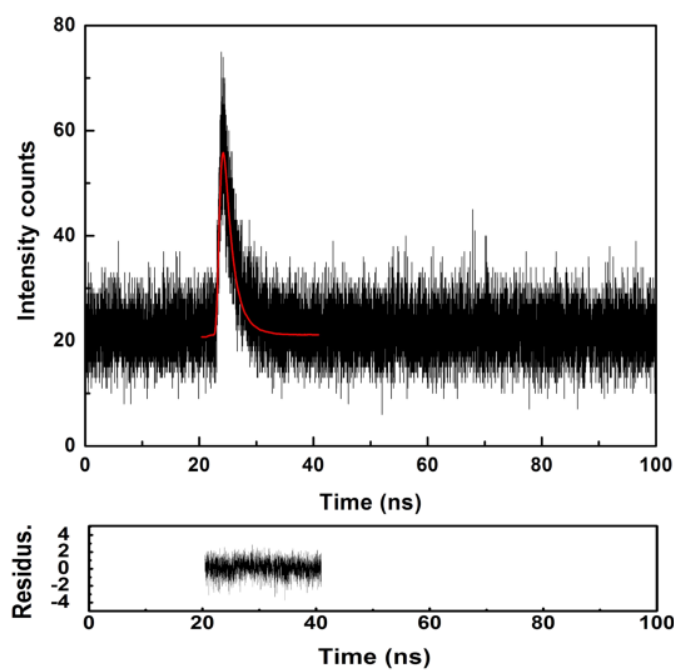

LmrR_W96
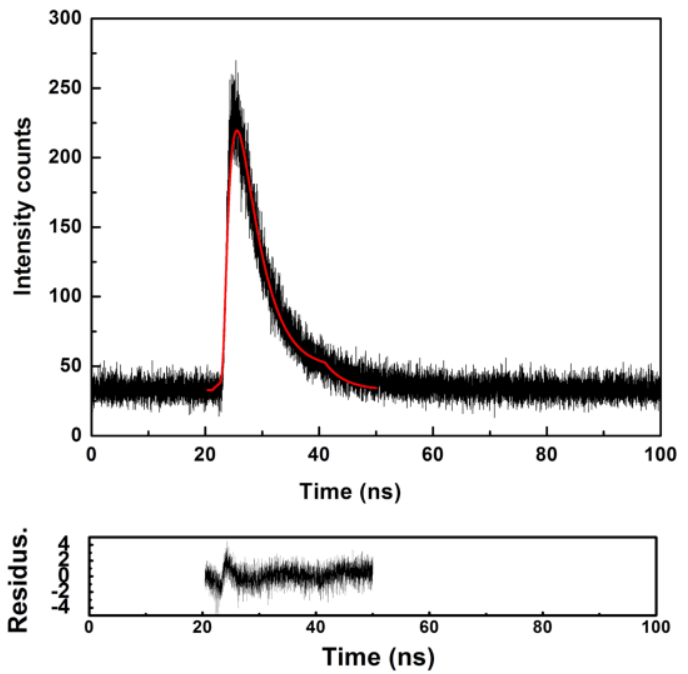

LmrR_W96+PplX
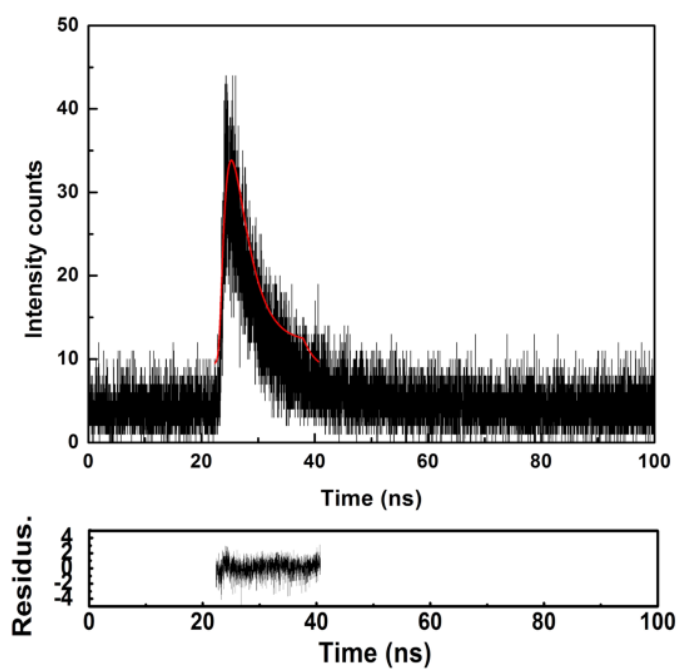
LmrR_W96+Bodipy
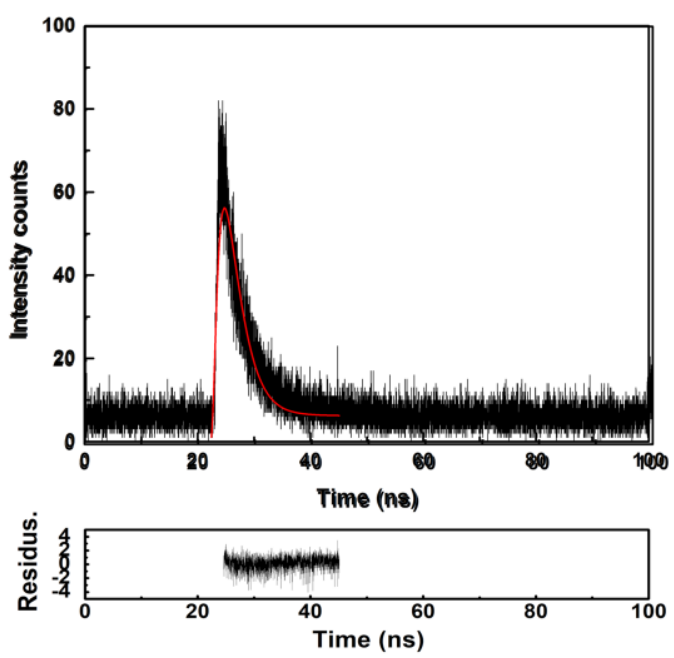

LmrR_W96+RBF
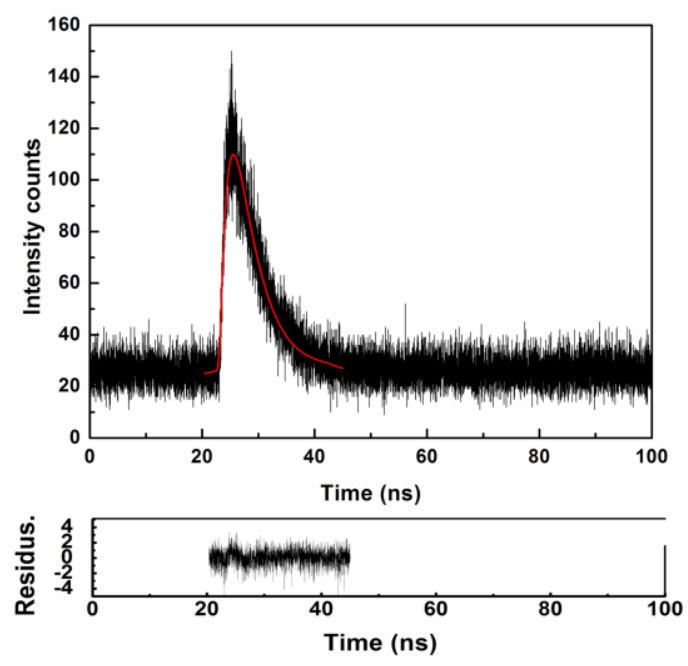

LmrR_W96+EY
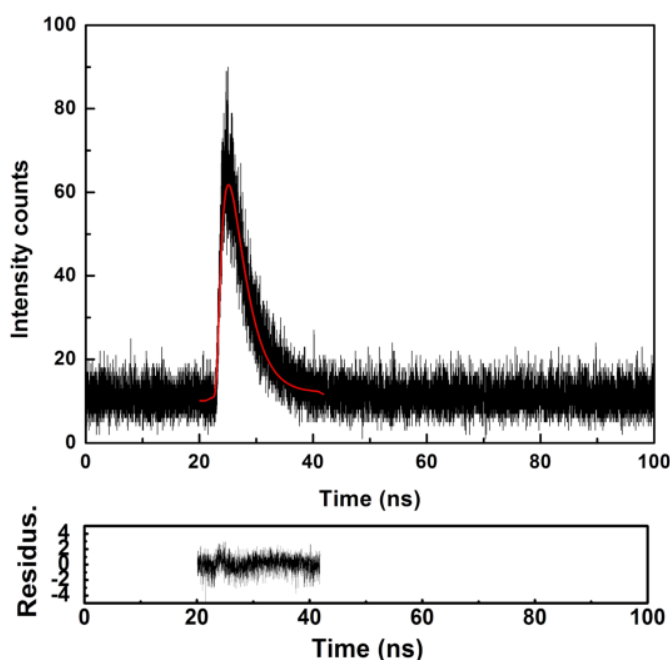

LmrR_W96+Rh6G
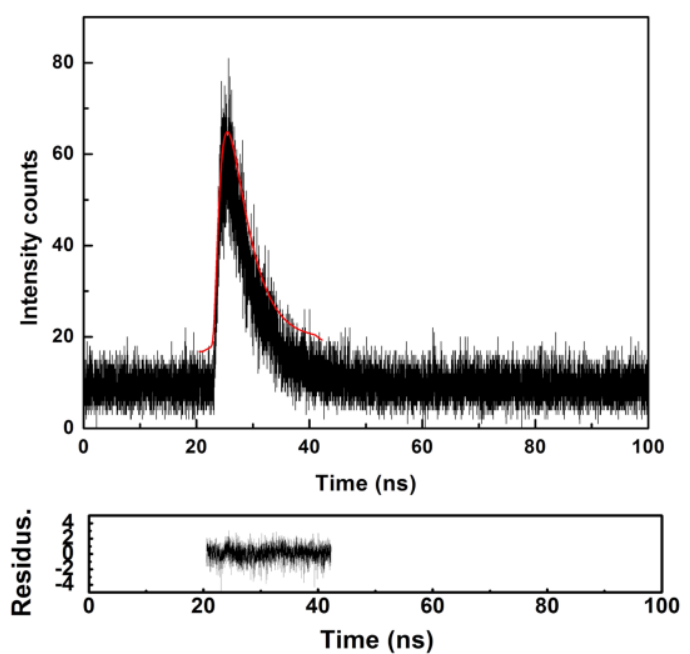

Figure S7. Tryptophan fluorescence lifetime decays for LmrR_W96 and LmrR_W96 in the presence of the dyes. Red lines indicated fit. 

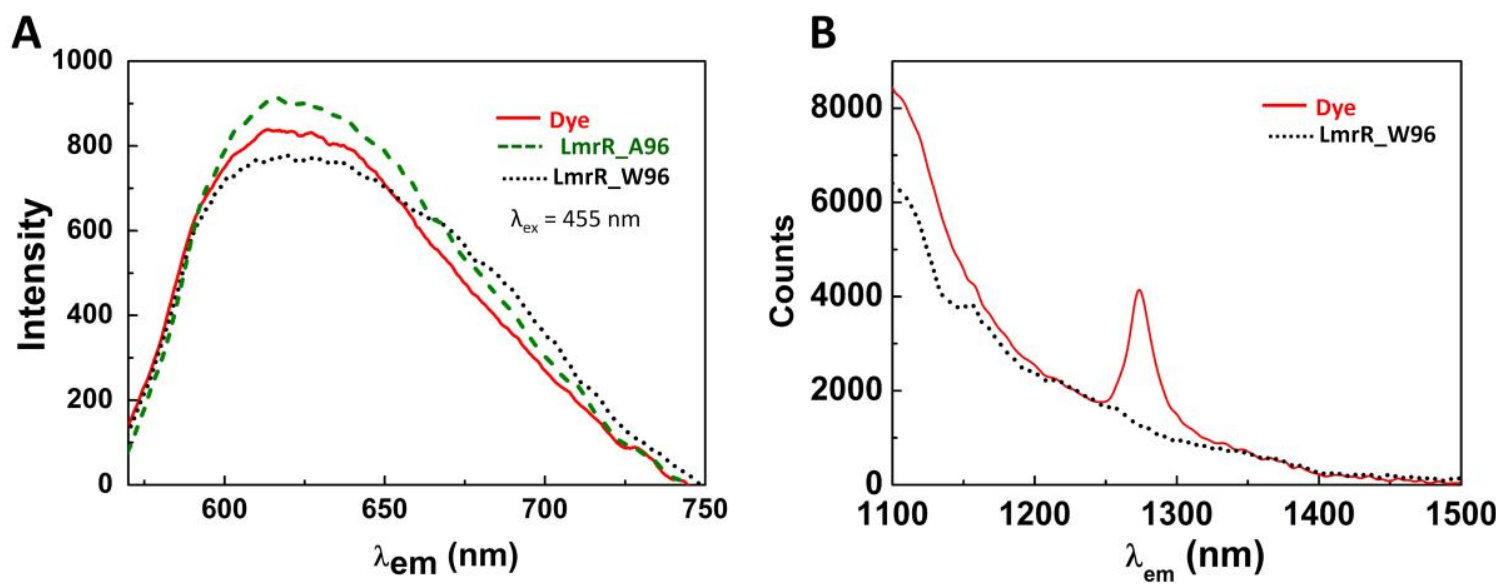

Figure 58. (a) Absorbance corrected $\left[\mathrm{Ru}(\mathrm{bpy})_{3}\right]^{2+}$ emission in phosphate buffer $\mathrm{pD} 8$ (red line), in the presence of LmrR_A96 (green dashed line) and in the presence of LmrR_W96 (black dotted line). $\lambda_{\text {ex }}=$ $455 \mathrm{~nm}$. (b) NIR emission spectra showing ${ }^{1} \mathrm{O}_{2}$ phosphorescence generated by excitation of $\left[\mathrm{Ru}(\mathrm{bpy})_{3}\right]^{2+}\left(\lambda_{\mathrm{ex}}=455 \mathrm{~nm}\right.$ ) in $\mathrm{D}_{2} \mathrm{O}$ (phosphate buffer pD 8) (red line) and in the presence of LmrR_W96 (black dotted line). The slopping background is due to emission from $\left[\mathrm{Ru}(\mathrm{bpy})_{3}\right]^{2+}$.
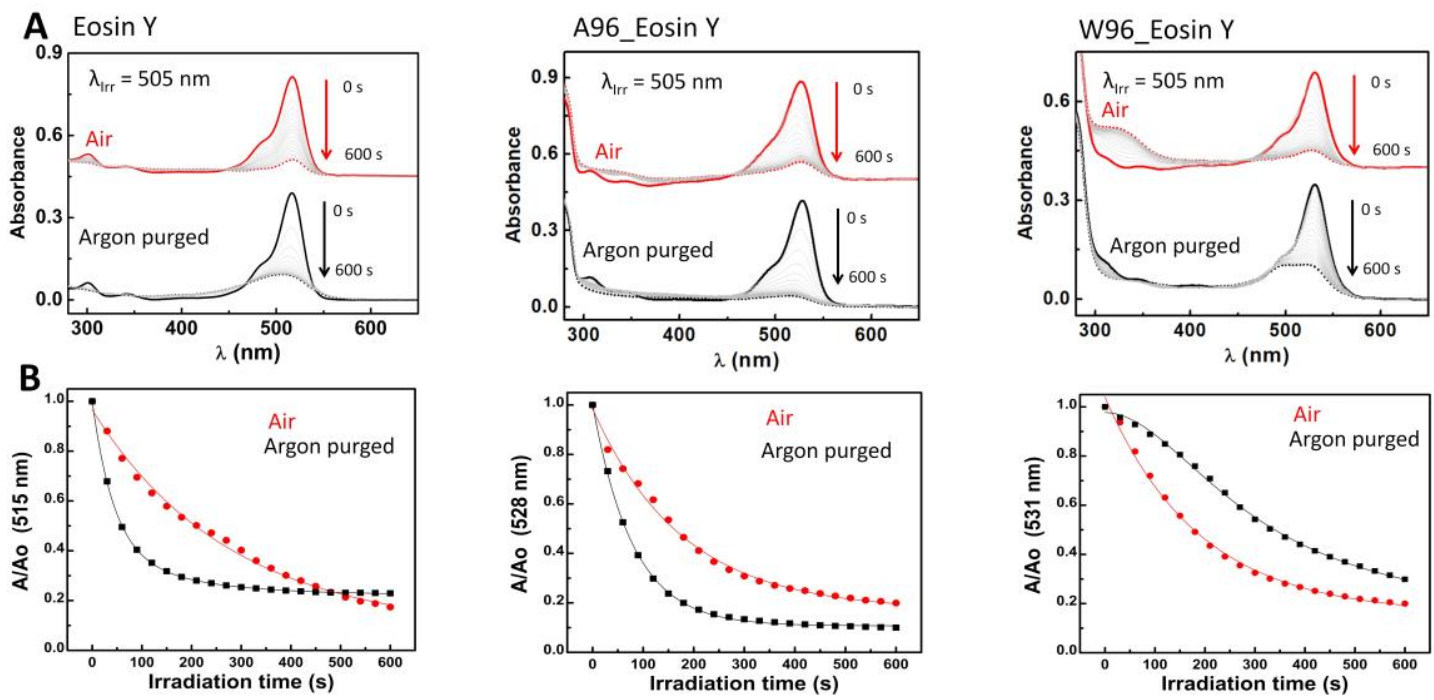

Figure S9. (a) Change in absorbance upon irradiation of eosin $Y$ from 0 to $600 \mathrm{~s}$ in the presence (red) and absence (black) of $\mathrm{O}_{2}$ (left), in the presence of LmrR_A96 (middle) and in the presence of LmrR_W96 (right). The initial spectrum (solid line); final spectrum (dashed line); grey lines are spectra recorded at $30 \mathrm{~s}$ intervals during continuous irradiation. (b) Absorbance with respect to initial absorbance at the maximum absorbance in the visible region of Eosin $\mathrm{Y}$ over time in the presence (red) and absence (black) of $\mathrm{O}_{2}$. Solid lines (black and red) are the fits using a model. Conditions: $4 \mu \mathrm{M}$ eosin $\mathrm{Y}$ in $50 \mathrm{mM}$ potassium phosphate buffer pD 8 prepared in $\mathrm{D}_{2} \mathrm{O}$. LmrR_A96 and LmrR_W96 are $16 \mu \mathrm{M}$ (1:4 dye:protein ratio). 

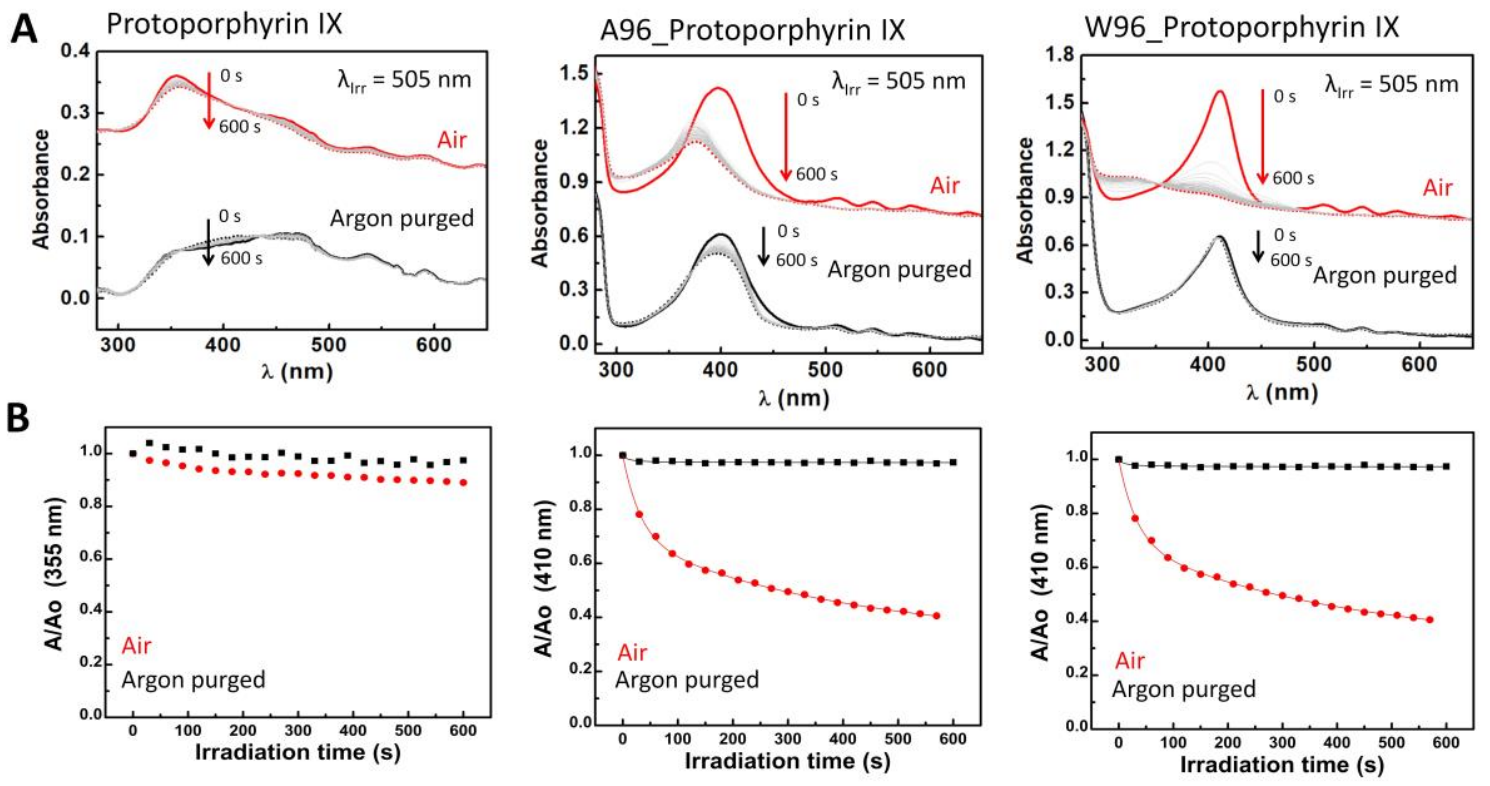

Figure S10. (a) Change in absorbance upon irradiation of protoporphyrin IX from 0 to $600 \mathrm{~s}$ in the presence (red) and absence (black) of $\mathrm{O}_{2}$ (left), in the presence of LmrR_A96 (middle) and in the presence of LmrR_W96 (right). The initial spectrum (solid line); final spectrum (dashed line); grey lines are spectra recorded at $30 \mathrm{~s}$ intervals during continuous irradiation. (b) Absorbance with respect to initial absorbance at the maximum absorbance in the visible region of protoporphyrin IX over time in the presence (red) and absence (black) of $\mathrm{O}_{2}$. Solid lines (black and red) are the fits using a model. Conditions: $10 \mu \mathrm{M}$ protoporphyrin IX in $50 \mathrm{mM}$ potassium phosphate buffer pD 8 prepared in $\mathrm{D}_{2} \mathrm{O}$. LmrR_A96 and LmrR_W96 are $40 \mu \mathrm{M}$ (1:4 dye:protein ratio).
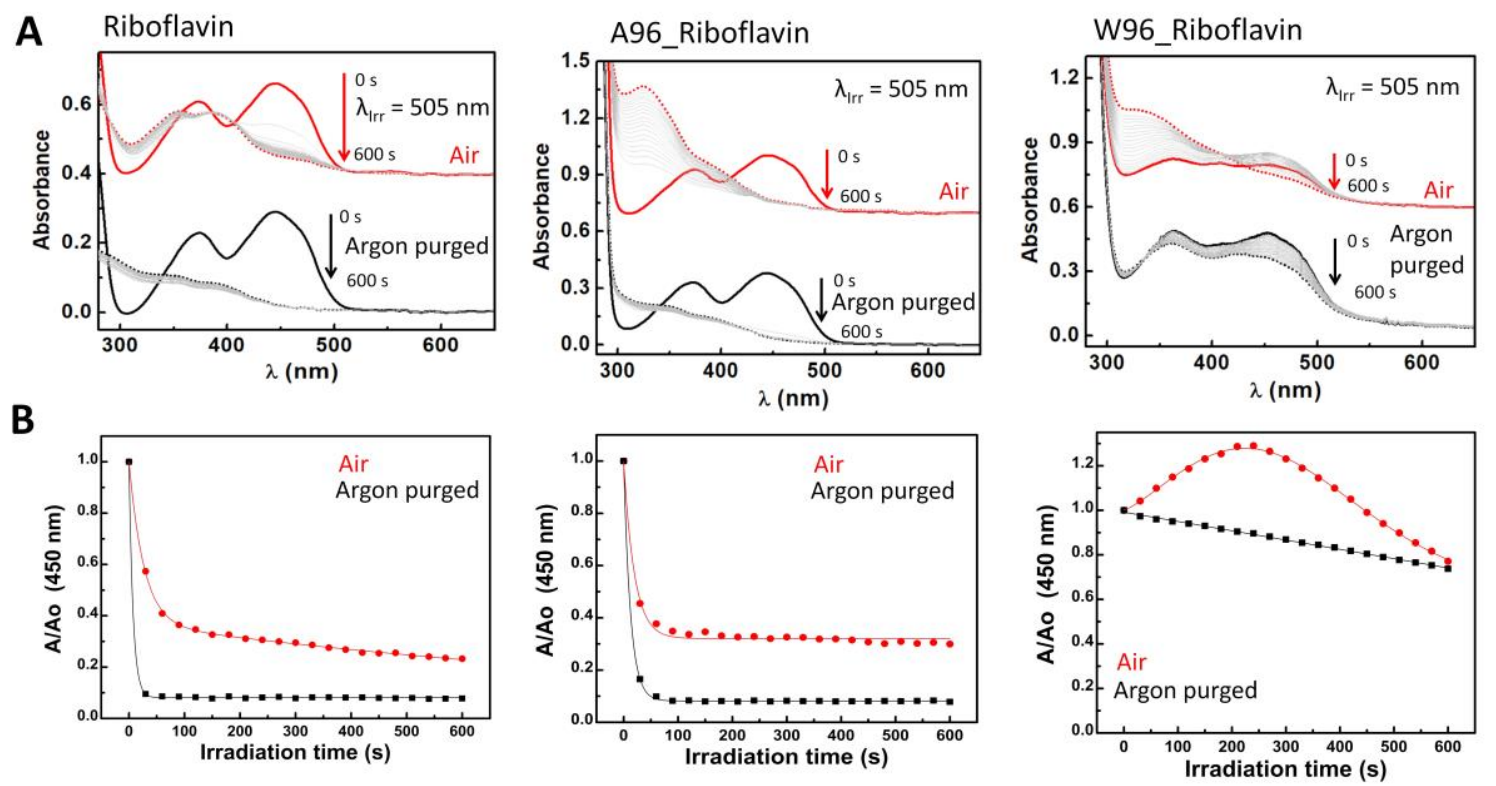

Figure S11. (a) Change in absorbance upon irradiation of riboflavin from 0 to $600 \mathrm{~s}$ in the presence (red) and absence (black) of $\mathrm{O}_{2}$ (left), in the presence of LmrR_A96 (middle) and in the presence of LmrR_W96 (right). The initial spectrum (solid line); final spectrum (dashed line); grey lines are spectra recorded at $30 \mathrm{~s}$ intervals during continuous irradiation. (b) Absorbance with respect to initial absorbance at the maximum absorbance in the visible region of riboflavin over time in the presence 
(red) and absence (black) of $\mathrm{O}_{2}$. Solid lines (black and red) are the fits using a model. Conditions: 30 $\mu \mathrm{M}$ riboflavin in $50 \mathrm{mM}$ potassium phosphate buffer pD 8 prepared in $\mathrm{D}_{2} \mathrm{O}$. LmrR_A96 and LmrR_W96 are $120 \mu \mathrm{M}$ (1:4 dye:protein ratio).

Table S1. Absorbance maximum in the visible region, fluorescence maximum and Stokes shift of the dyes in solution (red), in the presence of LmrR_W96A (green) and in the presence of LmrR_W96 (black).

\begin{tabular}{|l|l|l|l|l|l|l|l|l|l|}
\hline & \multicolumn{3}{|c|}{$\begin{array}{c}\text { Absorbance maximum } \\
\text { in the visible region } \\
\text { (nm) }\end{array}$} & \multicolumn{2}{c|}{$\begin{array}{c}\text { Fluorescence maximum } \\
\text { (nm) }\end{array}$} & \multicolumn{3}{c|}{ Stokes shift } \\
\hline & water & W96A & W96 & water & W96A & W96 & water & W96A & W96 \\
\hline Tryptophan & - & 280 & 280 & - & 312 & 312 & - & 32 & 32 \\
\hline RB & 549 & 559 & 563 & 565 & 573 & 572 & 16 & 14 & 9 \\
\hline PpIX & 376 & 398 & 411 & 621 & 637 & 633 & 245 & 230 & 222 \\
\hline Bodipy & 497 & 501 & 504 & 506 & 507 & 509 & 9 & 6 & 5 \\
\hline Eosin Y & 515 & 526 & 531 & 537 & 538 & 539 & 22 & 12 & 8 \\
\hline RBF & 444 & 444 & 454 & 526 & 526 & 526 & 82 & 82 & 72 \\
\hline Rh6G & 527 & 527 & 537 & 551 & 551 & 551 & 24 & 24 & 13 \\
\hline
\end{tabular}

Table S2. Fluorescence lifetime data in the presence of the quencher.

\begin{tabular}{l|l} 
Protein & Lifetime (ns) \\
\hline LmrR_W96 & 3.5 \\
LmrR_W96+PpIX & 2.4 \\
LmrR_W96+RB & 0.3 \\
LmrR_W96+EY & 2.4 \\
LmrR_W96+Rh6G & 3.3 \\
LmrR_W96+RBF & 3.7 \\
LmrR_W96+Bodipy & 2.9
\end{tabular}

Table S3. Ground state and exited state reduction $\left(\mathrm{E}_{\text {red }}\right)$ and oxidation $\left(\mathrm{E}_{\mathrm{ox}}\right)$ potentials of dyes. $\mathrm{E}_{\mathrm{oo}}$ is the energy gap between the zeroth vibrational level of the ground and excited states in the presence of LmrR_W96.

\begin{tabular}{|c|c|c|c|c|c|}
\hline & $\begin{array}{c}\mathrm{E}_{\mathrm{oo}} \\
(\mathrm{eV})\end{array}$ & $\begin{array}{c}\mathrm{E}_{\text {red }} \\
(\mathrm{V} \text { vs SHE) }\end{array}$ & $\begin{array}{c}\mathrm{E}_{\text {red }}^{*} \\
(\mathrm{~V} \text { vs SHE) }\end{array}$ & $\begin{array}{c}\mathrm{E}_{\text {oxi }} \\
(\mathrm{V} \text { vs SHE) }\end{array}$ & $\begin{array}{c}\mathrm{E}_{\text {oxi }} \\
\text { (V vs SHE) }\end{array}$ \\
\hline Rose bengal & 2.18 & -0.54 & 1.64 & 1.33 & -0.85 \\
\hline Protoporphyrin IX & 2.00 & -0.23 & 1.77 & $\mathrm{x}$ & $\mathrm{x}$ \\
\hline Bodipy & 2.45 & -1.20 & 1.25 & 1.28 & -1.17 \\
\hline Eosin Y & 2.32 & -1.06 & 1.26 & 0.78 & -1.54 \\
\hline Riboflavin & 2.49 & -1.22 & 1.27 & $\mathrm{x}$ & $\mathrm{x}$ \\
\hline Rhodamine 6G & 2.28 & -0.71 & 1.57 & 1.63 & -0.65 \\
\hline Tryptophan & & & & 1.09 & \\
\hline
\end{tabular}


Table S4. Fitting of changes in visible absorbance over time under continuous irradiation for rose bengal in $50 \mathrm{mM}$ phosphate buffer pD 8, in the presence of LmrR_A96 and in the presence of LmrR_W96. Models used are indicated in the table. Protein:dye ratio 1:4.

\begin{tabular}{|c|c|c|}
\hline & \multicolumn{2}{|c|}{ Rose bengal } \\
\hline & Air & Argon purged \\
\hline & $Y=A^{*} \exp (-K x)+Y_{0}$ & $Y=A^{*} \exp (-K x)+Y_{0}$ \\
\hline $\mathrm{K}\left(\mathrm{s}^{-1}\right)$ & $2.4 \times 10^{-3}$ & $2.6 \times 10^{-3}$ \\
\hline A & 0.8 & 0.6 \\
\hline $\mathrm{Y}_{\mathrm{o}}$ & 0.2 & 0.4 \\
\hline \multirow[t]{4}{*}{ R-Sqr } & 0.99 & 0.99 \\
\hline & \multicolumn{2}{|c|}{ A96_Rose bengal } \\
\hline & Air & Argon purged \\
\hline & $Y=A /\left(1+k_{1} x\right)-K_{2} x+Y_{0}$ & $Y=Y_{0}-K_{1} X$ \\
\hline $\mathrm{K}_{1}\left(\mathrm{~s}^{-1}\right)$ & 0.9 & $2.8 \times 10^{-4}$ \\
\hline$K_{2}\left(s^{-1}\right)$ & $2.9 \times 10^{-4}$ & - \\
\hline \multicolumn{3}{|l|}{$\mathrm{A}$} \\
\hline $\mathrm{Y}_{0}$ & 0.8 & 1 \\
\hline \multirow[t]{4}{*}{ R-Sqr } & 0.99 & 0.99 \\
\hline & \multicolumn{2}{|c|}{ W96_Rose bengal } \\
\hline & Air & Argon purged \\
\hline & $Y=A /\left(1+k_{1} x\right)-K_{2} x+Y_{0}$ & $Y=Y_{0}-K_{1} X$ \\
\hline $\mathrm{K}_{1}\left(\mathrm{~s}^{-1}\right)$ & 0.05 & $9.3 \times 10^{-5}$ \\
\hline$K_{2}\left(s^{-1}\right)$ & $1.7 \times 10^{-4}$ & - \\
\hline \multicolumn{3}{|l|}{$A$} \\
\hline $\mathrm{Y}_{\mathrm{o}}$ & 0.5 & 0.99 \\
\hline R-Sqr & 0.99 & 0.99 \\
\hline
\end{tabular}

Table S5. Fitting of changes in visible absorbance over time under continuous irradiation for bodipy in 50 mM phosphate buffer pD 8, in the presence of LmrR_A96 and in the presence of LmrR_W96. Models used are indicated in the table. Protein:dye ratio 1:4.

\begin{tabular}{|c|c|}
\hline & Bodipy \\
\hline & Air \\
\hline & $\mathrm{Y}=\mathrm{A}^{*} \exp (-\mathrm{Kx})+\mathrm{Y}_{\circ}$ \\
\hline $\mathrm{K}\left(\mathrm{s}^{-1}\right)$ & $2.7 \times 10^{-3}$ \\
\hline $\mathrm{A}$ & 1 \\
\hline $\mathrm{Y}_{\circ}$ & 0 \\
\hline $\mathrm{R}-\mathrm{Sqr}$ & 0.99 \\
\hline & A96_Bodipy \\
\hline & Air \\
\hline & $\mathrm{Y}=\mathrm{A}^{*} \exp (-\mathrm{Kx})+\mathrm{Y}_{\mathrm{o}}$ \\
\hline $\mathrm{K}\left(\mathrm{s}^{-1}\right)$ & $2.7 \times 10^{-3}$ \\
\hline $\mathrm{A}$ & 1 \\
\hline $\mathrm{Y}_{\circ}$ & 0 \\
\hline $\mathrm{R}-\mathrm{Sqr}$ & 0.99 \\
\hline
\end{tabular}


Table S6. Protein:dye ratio 1:4. Fitting of changes in visible absorbance over time under continuous irradiation for Eosin $Y$ in $50 \mathrm{mM}$ phosphate buffer pD 8, in the presence of LmrR_A96 and in the presence of LmrR_W96. Models used are indicated in the table. Protein:dye ratio 1:4.

\begin{tabular}{|c|c|c|}
\hline & \multicolumn{2}{|c|}{ Eosin Y } \\
\hline & Air & Argon purged \\
\hline & $Y=A^{*} \exp (-K x)+Y_{0}$ & $Y=A^{*} \exp (-K x)+Y_{0}$ \\
\hline$K\left(s^{-1}\right)$ & $3.6 \times 10^{-3}$ & $1.6 \times 10^{-2}$ \\
\hline $\mathrm{A}$ & 0.9 & 0.8 \\
\hline$Y_{0}$ & 0.08 & 0.24 \\
\hline \multirow[t]{4}{*}{ R-Sqr } & 0.99 & 0.99 \\
\hline & \multicolumn{2}{|c|}{ A96_Eosin Y } \\
\hline & Air & \begin{tabular}{|l|} 
Argon purged \\
\end{tabular} \\
\hline & $Y=A^{*} \exp (-K x)+Y_{0}$ & $Y=A^{*} \exp (-K x)+Y_{0}$ \\
\hline$K\left(s^{-1}\right)$ & $5.6 \times 10^{-3}$ & $1.1 \times 10^{-2}$ \\
\hline$A$ & 0.8 & 0.9 \\
\hline$Y_{0}$ & 0.2 & 0.1 \\
\hline \multirow[t]{4}{*}{ R-Sqr } & 0.99 & 0.99 \\
\hline & \multicolumn{2}{|c|}{ W96_Eosin Y } \\
\hline & Air & Argon purged \\
\hline & $Y=A^{*} \exp (-K x)+Y_{0}$ & $Y=A /\left(1+(K x)^{\wedge} \beta\right)+Y_{0}$ \\
\hline$K\left(\mathrm{~s}^{-1}\right)$ & $5.3 \times 10^{-3}$ & $3.3 \times 10^{-3}$ \\
\hline A & 0.9 & 0.9 \\
\hline$Y_{0}$ & 0.2 & 0.1 \\
\hline R-Sqr & 0.99 & 0.99 \\
\hline
\end{tabular}

Table S7. Fitting of changes in visible absorbance over time under continuous irradiation for protoporphyin IX in $50 \mathrm{mM}$ phosphate buffer pD 8, in the presence of LmrR_A96 and in the presence of LmrR_W96. Models used are indicated in the table. Protein:dye ratio 1:4.

\begin{tabular}{|c|c|}
\hline & A96_Protoporphyrin IX \\
\hline & Air \\
\hline & $\mathrm{Y}=\mathrm{A}^{*} \exp (-\mathrm{Kx})+\mathrm{Y}_{\circ}$ \\
\hline $\mathrm{K}\left(\mathrm{s}^{-1}\right)$ & $1.8 \times 10^{-3}$ \\
\hline $\mathrm{A}$ & 0.5 \\
\hline $\mathrm{Y}_{\circ}$ & 0.5 \\
\hline $\mathrm{R}-\mathrm{Sqr}$ & 0.99 \\
\hline & W96_protoporphyin IX \\
\hline & Air \\
\hline & $\mathrm{Y}=\mathrm{A}_{1}{ }^{*} \exp \left(-\mathrm{K}_{1} \mathrm{x}\right)+\mathrm{A}_{2}{ }^{*} \exp \left(-\mathrm{K}_{2} \mathrm{X}\right)+\mathrm{Y}_{\circ}$ \\
\hline $\mathrm{K}_{1}\left(\mathrm{~s}^{-1}\right)$ & $5.8 \times 10^{-2}$ \\
\hline $\mathrm{K}_{2}\left(\mathrm{~s}^{-1}\right)$ & $4.1 \times 10^{-3}$ \\
\hline $\mathrm{A}_{1}$ & 0.6 \\
\hline $\mathrm{A}_{2}$ & 0.2 \\
\hline $\mathrm{Y}_{0}$ & 0.2 \\
\hline $\mathrm{R}-\mathrm{Sqr}$ & 0.99 \\
\hline
\end{tabular}


Table S8. Protein:dye ratio 1:4. Fitting of changes in visible absorbance over time under continuous irradiation for riboflavin in $50 \mathrm{mM}$ phosphate buffer pD 8, in the presence of LmrR_A96 and in the presence of LmrR_W96. Models used are indicated in the table. Protein:dye ratio 1:4.

\begin{tabular}{|c|c|c|}
\hline & \multicolumn{2}{|l|}{ Riboflavin } \\
\hline & Air & Argon purged \\
\hline & $Y=A_{1} * \exp \left(-K_{1} x\right)+A_{2} * \exp \left(-K_{2} x\right)+Y_{0}$ & $Y=A^{*} \exp (-K x)+Y_{0}$ \\
\hline$K_{1}\left(s^{-1}\right)$ & $3.7 \times 10^{-2}$ & $1.4 \times 10^{-1}$ \\
\hline $\mathrm{K}_{2}\left(\mathrm{~s}^{-1}\right)$ & $8.1 \times 10^{-4}$ & $\mathrm{X}$ \\
\hline$A_{1}$ & 0.6 & 0.9 \\
\hline$A_{2}$ & 0.4 & $\mathrm{x}$ \\
\hline $\mathrm{Y}_{\mathrm{o}}$ & 0 & 0.1 \\
\hline \multirow[t]{4}{*}{ R-Sqr } & 0.99 & 0.99 \\
\hline & \multicolumn{2}{|l|}{ A96_Riboflavin } \\
\hline & Air & Argon purged \\
\hline & $Y=A^{*} \exp (-K x)+Y_{0}$ & $Y=A^{*} \exp (-K x)+Y_{0}$ \\
\hline$K\left(s^{-1}\right)$ & $5.1 \times 10^{-2}$ & $7.9 \times 10^{-2}$ \\
\hline A & 0.9 & 0.7 \\
\hline $\mathrm{Y}_{\mathrm{o}}$ & 0.1 & 0.3 \\
\hline R-Sqr & 0.99 & 0.99 \\
\hline
\end{tabular}

\section{Emission lifetime decay data.}

Table S9. Fluorescence decay lifetime of tryptophan in the presence of quencher.

\begin{tabular}{l|l}
\multicolumn{1}{l|}{ Protein } & Lifetime (ns) \\
\hline LmrR_W96 & 3.5 \\
LmrR_W96+PpIX & 2.4 \\
LmrR_W96+RB & cross-correlated \\
LmrR_W96+EY & 2.4 \\
LmrR_W96+Rh6G & 3.3 \\
LmrR_W96+RBF & 3.7 \\
LmrR_W96+Bodipy & 2.9 \\
LmrR_W96+[Ru(bpy $\left.)_{3}\right]^{2+}$ & 3.1
\end{tabular}

Table S10. Fluorescence decay lifetime of dyes in solution and in the presence of LmrR_W96.

\begin{tabular}{|c|l|}
\hline & $\begin{array}{c}\text { Fluorescence lifetime } \\
\text { (ns) }\end{array}$ \\
\hline Protoporphyrin IX & \\
\hline Water & cross-correlated \\
\hline LmrR_W96 & 8.2 \\
\hline \hline Methanol & 14.2 \\
\hline \hline DMSO & 22.9 \\
\hline Rose Bengal & \\
\hline
\end{tabular}




\begin{tabular}{|c|l|}
\hline Water & cross-correlated \\
\hline LmrR_W96 & cross-correlated \\
\hline Methanol* & 0.55 \\
\hline Acetonitrile & 2.3 \\
\hline Eosin Y & \\
\hline Water & 1.12 \\
\hline \hline LmrR_W96 & 1.8 \\
\hline Ethanol & 3.3 \\
\hline DMSO & 3.8 \\
\hline Rhodamine 6G & \\
\hline \hline water & 4.1 \\
\hline LmrR_W96 & 3.8 \\
\hline DMSO & 3.8 \\
\hline Riboflavin & \\
\hline Water & 4.5 \\
\hline LmrR_W96 & 4.5 \\
\hline Acetonitrile & 4.7 \\
\hline Bodipy & \\
\hline \hline Water & 1.7 \\
\hline \hline LmrR_W96 & 4.7 \\
\hline Methanol & 3.7 \\
\hline
\end{tabular}

*D. Huppert, S. D. Rand, A.H. Reynolds and P.M. Rentzepis, J. Chem. Phys. 1982, 77, 1214-1224 